\title{
تقييم المعرفة المالية بالعملات المشفرة
}

\section{دراسة استطلاعية لاراء عينة من مدراء الشركات والملكاتب FOREX في اقليم كودستان

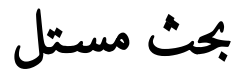

\author{
أ.مد. شفان اممد محم، قسم إدارة الأعمال، كلية الإدارة والاقتصاد -جامعة نوروز ، أقليم كوردستان العراق

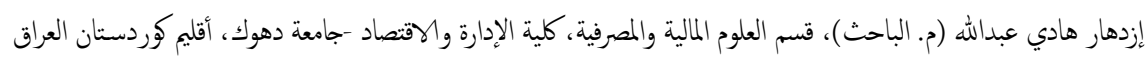

تهدف الدراسة الحالية إلى التعرف على العملات المشفرة وانواعها وخصائصها ومدى إدراك المتعملين بتبادل العملات في الإقليم بالعملات المشفرة من خلال دراسة أستطلاعية لآراء عينة من المدراء واصحاب الشركات / Forex وتبادل العملات في اقليم كوردستان العراق للعملات المشفرة من خلال تصميم أستارة أستبانة والتي تضمنت عدداً من العبارات الأساسية للاراسة ومت الإجابة

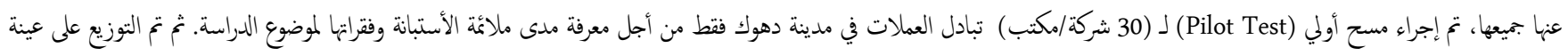

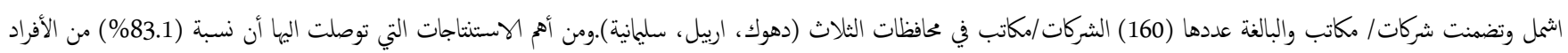
المبحوثين للأقليم ليس لديم اي معرفة مالية بالية العمل بالعملات المشفرة وانواعها وكيفية أستخداها وطريقة تداولها مقابل (16.9\%) لدهم معرفة مالية بآلية التداول بالعملات المشفرة ، تبين من نتأج التعليل نسبة (100\%) عدم تداول العملات المشفرة من الشركات والمكاتب للعينة المبحوثة سواءأكانت طريقة التداول المباشرة او تداول غير مباشر . وفي ضوء تلك الكستناجات وضعت الدراسة

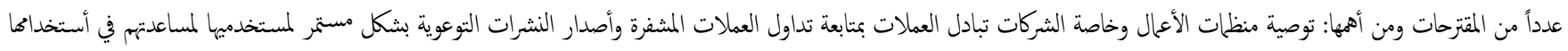

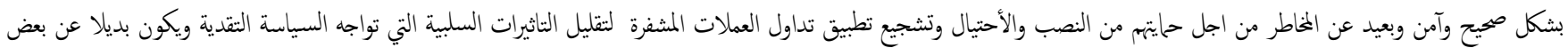
العملات

الكلمات المفتاحية: العملات المشفرة، المعرفة المالية ، التعدين ، تبادل العملات، القرصنة.

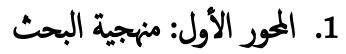

إما في العراق فقد حذر البنك المركزي العراقي المواطنين العراقيين بنداول عملة 1.1 Bitcoin البنك المركزي العراقي في بيان رسمي وتعميم بعدم أستخداما وأخضاع المتعاملين بها لأحكام قانون غسل الأموال رث (39) لسنة (2015) والقوانين ذات العلاقة بهذا

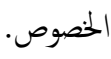
بناء على ما تقدم يمكن ان نطرح السؤال النالي للتعبير عن مشكلة الدراسة ماهو مستوى المعرفة المالية والرؤية المستقبلية للعملات المشفرة في الأقليم . قبل ظهور العملة المشفرة كانت الجهة الوحيدة القادرة على اصدار العملة هي البنوك المركزية والتي بدورها لم تسعى الى تحقيق الربح بل هدفها احداث توازن بين عرض

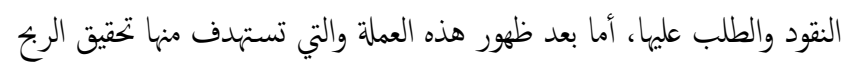
هناك تخوف من ان هذه العملة تخلق مشكلة في النظام النقدي العالمي، بينت دراسة ( Ciaian, Rajcaniova, \& Kancs, 2015) تحديده بثلاثة أشياء، وهي التفاعل بين العرض والطلب، الجاذبية للمستثمرين، و ظروف الاقتصاد الكلي والتطورات المالية. امكانية وقدرة العملة المشفرة في تأثير على الاقتصاد العالمي بايجابياتها وسلبياتها، ومن خلال هذا الدراسة نحاول ان نسلط الضوء على ماهية هذه العمة المشفرة وماهي محدداتها ومخاطرها وخصائصها. بالإضافة الى دراسة مدى إمكانية وجود عملة يزداد ججم الأستثارات في العملات المشفرة يومياً بعد يوم حيث تجاوز جحم القمة السوقية اليومية (181) مليار دولار يومياً، وتجاوز سعر البتكوين الواحد حاجز

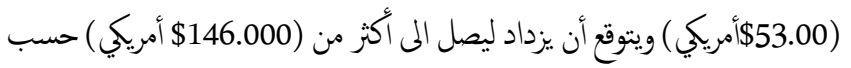
الكترونية كوردية مستقبلا وفي ضوء ذلك كون أن العديد من الحكومات، الشركات، توقعات وخبرات Forbey.com) JP Morgan). 


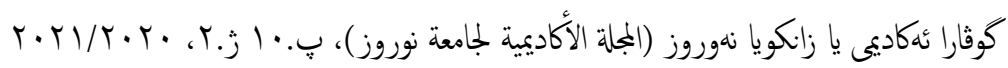

- - معرفة مدى إدراك المتعاملين بتبادل العملات في الإقليم بالعملات المشفرة.

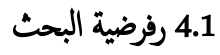

ينطلق البحث من فرضية عدم وجود ادراك للمتعاملين بالعملات الأجنبية في الإقليم

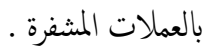

5.1

اعتمدت البحث المنهج الأستطلاعي

6.1 اساليب جمع المعلومات

يعتمد البحث الحالي في بناء الجانب النظري على مصادر من رسائل، أطاريه، دراسات، كتب وأساليب النحليل الأحصائي المتعلقة بموضوع البحث والاطلاع على مواقع العملات المشفرة وذلك انسجاما مع أحدث بجوث ودراسات التي تناول الموضوع الدراسة الحالية، اما في الجانب التطبيقي اعثمد البحث على أجابات أسئلة الأستارة الأستبانة التي وزعت على الشركات / مكاتب Forex التداول وتبادل

العملات في الأقليم للعملات المشفرة لمعرفة مدى إدراكمم بالعملات المشفرة .

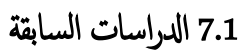

تمثل الدراسـات السـابقة العمق النظري للأفكار والمرتكزات الرئيسية لبناء هيكل الدراسة عامةً ومنهجيتها خاصةً، فضلاً عن أنها الميدان العملي لتطبيق تلك الأفكار ، واستكمالاً للإطار النظري الخاص بالدراسة. وترى الباحثة ضرورة استعراض عدد من الدراسـات النظرية والتطبيقية التي سـاهمت في رسم الأطر الهيكيلة والبنائية لمنهجية الدراسة حيث تم عن طريقها الاطلاع على ما قدمه الآخرون من دراسات وبحوث نظرية وميدانية ذات صـلة بموضـوع دراستنا الحالية وما توصـلت الية من اسـتناجات ومقترحات ومحاولة معرفة ما يككن اضـافنه، لكونها هي الأسـاس لأي دراسة لاحقة حيث اسيتمدت منها المفاهيم النظرية لتأطير الدراسة الحالية ـ وسيتم عرض اهم الدراسـات الأجنبية والعربية التي تيسرــلباحثة الحصــول عليها والتي

$$
\text { تناولت العملات المشفرة كما يلي : }
$$

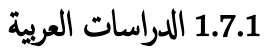

1. 1.1.7.1 دراســة : ( أبو صـلاح، 2018) ـ عنوان الدراسـة : العملات الرقية

\section{وملاقتها بالتجارة الإلكتونية : دراسة حالة دولة الأمارات العربية المتحدة (دي) .}

هدفت الدراسـة تحديد العلاقة بين العملات الرقية والتجارة الكككتونية لعدد من الشر_كات في دولة الأمارات للفترتين الممتدة (2000-2007) (2008-2017)، أهمية أســتخدام الحمافظ الرقية في تخزين العملات المشــفرة لما لها من اهمية في
والمؤسسات قد أصدرت عملة ألكترونية خاصة بها سواءاً كانت رقية أم مشفرة، يكن توضيح أهمية الدراسة من خلال النقاط التالية:

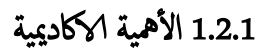

يكتسب البحث الحالي أهميها الكاديمية لإثارتها مواضيع ذات أهمية بالغة وحديثة في بيئة الاعمال، والعمليات المالية الدولية، المصارف والسياسية النقدية بالإضافة الى الاستثار في الأسواق المالية وتكوين محظة أستثراية، نظرا لقلة الدراسة الكاديمية عن موضوع العملات الرقية المشفرة وإمكانية توضيح الجانب النظري الفلسفي من خلال التعرف على العوامل التي تؤثر في سعر العملات الرقية المشفرة سواء عوامل اقتصادية ام عوامل متعلقة بنكلة التنقيب عن العملات الرقية المشفرة يعد من الأهمية بككن دراستها. ونظرا لقلة الدراسات الكاديمية التي ربطت العملات

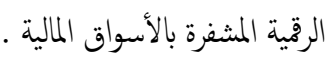
بالإضافة الى محاولة التعرف على معوقات عدم وجود عملة رقمية مشفرة كوردية لحد كنابة هذه الدراسة، ودراسة احتالية وجودها من عدها في المستقبل بالإضافة الى لى مدى إدراك المتعاملين بتبادل العملات الاجنبية للعملات المشفرة.

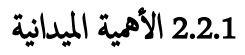

يكتسب البحث الحالي أهيتها الميدانية من منطلق ان الاقتصاد العراقي بشكل عام يعتمد على مصدر واحد رئيسي للدخل وهو النفط والغاز والذي يمثل حوالي 93\% من إيرادات الدولة العراقية، وينطبق نفس الحالة على إقليم كوردستان العراق وربما يزداد صعوبة نظرا للتقلبات التي تحصل بين حكومة الإقليم في أربيل والحكومة

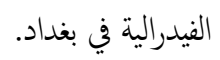
تعد هذه الدراسة كمة للعديد من الجهات مثل وشركات ومكاتب تبادل العملات الأجنبية والمستثمرين الصغار المتداولين في مكاتب Forex لتوسيع آفاق الأستثمارات للعملات المشفرة في المستقبل بعد ان يتم تميئة الاطار التشريعي والقانوني لها، للأكادييين والباحثين في تخصص الإدارة المالية، العلوم المالية والمصرفية، والمحاسبة،

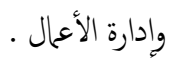

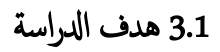
هبدف البحث الحالي لتحقيق الأهداف التالية: - - التعرف على العملات المشفرة وانواعها وخصائصها . - التعرف على اهم محددات العملات المشفرة وبالأخص تكاليف التنقيب عنها. 


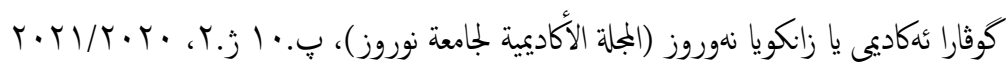

زيادة الوعي والثــفافية ، وقد يؤدي في النهاية إلى تحقيق ذلك هذا الســوق أقل تقلبًا وأكثر قابلية للتنبؤ به وأقل عرضة لأي تلاعب.

(Holovatiuk: 2020) 2.2.7.1 عنوان الدراسة

\section{Cryptocurrencies as an asset class in portfolio optimization}

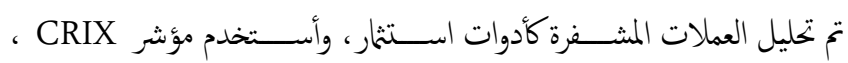
اســتخدمت بيانات يومية من 2014/8/1 الى 2019/7/17. من منصــة جامعة هومبولت في برلين بالتعاون مع إدارة جامعة سنغافورة، تبين أن أصول التشفير لها فوائد التنويع للمحفظة التقليدية، كما أن العملات المشـفرة في المحفة الأستـثارية تؤدي إلى أداء متفوق حسب معدل الخخاطر .حيث أضافت آلية التحسين 1.9٪ من العملات المشــفرة إلى المحافظ ذات المراكز الطوياة فقط و 2.8\% للمحافظ طويلة وقصـــرة المدى .كما أن هناكـ زيادة في الأداء بعد إدراج مؤشر العملات المشفرة لمفظة الأصول التقليدية لفترة المواقف طوياة فقط ، نسبة Sharpe من الحد الأدنى- زادت محفظة الفروق بنسبة 3\% ، في حين زادت محفظة زيادة محفظة التماس بنسبة 10\% .لمحافظ مع كل من المراكز الطوية والقصيرة ، نسبة شارب

$$
\text { بنسبة 3\% و 7\% على التوالي . }
$$

\section{3eker et at,2019) 3نورن المراسة}

\section{Determinants of Cryptocurrency Price Movements}

ركزت هذه الدراسة على كيفية ثاثثير (أسعار الفائدة وأسواق الأسهم وأسعار الذهب) كوامل الخارجية و (المؤشرات بشكل أساسي على أنها شعبية وإضفاء الشرعية)

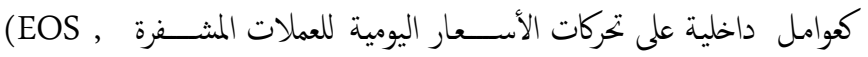
، Tether, Litecoin, Ethereum, Bitcoin). المسـجلة والمختلفة للفترة بين (1 أغسـطس ، 2017 - 3 أبريل 2019 ). أظهرت نتائج اختبار النكمل المشـترك بوجود علاقة تكامل عند مسـتوى 5\% في النموذج حيث يكون المتغير التابع فقط هو Tether، و لم يتم الثثور على علاقة مشـتركة

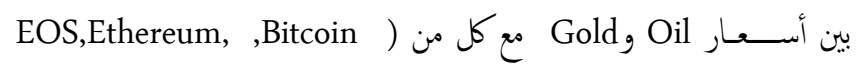
Liteconm, سبية أحادية الاتجاه بنسبة 10\% .مستوى الأهمية من Oil إلى Bitcoin ، من . Oil الى Litecoin EOS G Ethereum من Gitecoin
الحفاظ على الأموال بطريقة آمنة، واصــدار النشرـات التوعية بشـكل مسـتمر لمستخدميها من اجل أستخدامها بشكل لصحيح والآمن وبعياً عن الخخاطر .توصلت الدراســـة الى وجود علاقة ذات دلالة احصـــائية بين العملات الرقية والتجارة 1. الككترونية والسـبب في هذه النتيجة هو أن العملات المثـفرة هي حصيلة ثورة تكنولوجيا المعلومات المتطورة والمتســارعة، والمحافظ الرقية قد جاءت كتحكم رئسي- في أسـتقبال وصرف وتحويل العملات المثـفرة وكوسـيلة آمنة لحفظ العملات المشـفرة، في ضـوء النتائُ اقترحت الدراسـة الاهثمام بتطوير أسـتخدام التجارة الألكترونية عن طريق السـي نخو زيادة أنتشار العملات الرقية مع الأخذ بعين الاعتبار تجنب مخاطرها.

2.1.7.1 دراسة : (أحمد وآخرون، 2018) عنوان دراسة : (الكفاءة الأقتصادية اللعملات الأفتراضية المشفرة ، البتكوين أثموذجاً)

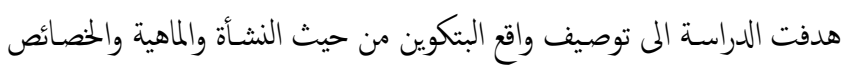

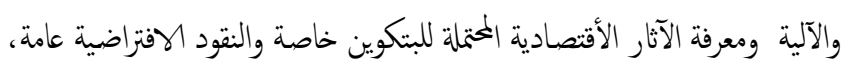
اتبعت الدراسة المنهج الوصفي لمعع المعلومات والمنهج التحليلي لمعرفة آثار أستخدام العملة على المستوى الأقتصادي، توصلت الدراسة المى أن العملة الأفتراضية عرضة للمخاطرة التي توثر في قيمها واسـتقرارها وعدم وجود تنظيم ورقابة لهذه العملات يؤدي الى ضياع الحقوق في حال اختلت الموازين العدل وتكونرهينة لقوى السوق والمضاربات، في ضوء النتائُ اقترحت الدراسة الى إجراء دراسة قياسية لمعرفة أثر تكثر وسائل الدفع الإلكترونية على معدلات التضخم وأدوات السياسة النقدية .

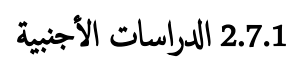

Financial عنوان الدراسة (Panos et al,2020): 1.2.7.1 Literacy and Attitudes to Cryptocurrencies تبحث هذه الدراسـة في الدور الهام لمحو الأمية المالية في تكوين المواقف فيا يتعلق

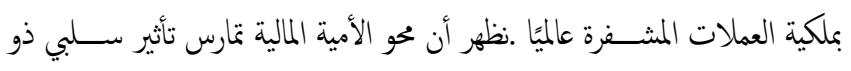
دلالة إحصــائية على احتالية امتلاك عملة. حيث يثبين أن الثقافة المالية تؤثر من حيث الحجم والأهمية .هذا يؤكده التأثير السـلبي الأكبر لمكون الخخاطر المالية للمالية تدبير معرفة القراءة والكتابة على الملكية وعلى نية امتلاك العملات المشــفرة في المستقبل. وهناك حاجة إلى بذل جحود لزيادة فهم الجمهور لجانب العرض وتكينه تحقيق في دوافع وحوافز جانب الطلب في السـوق لـــــالعملات الرقية .سيؤدي ذلك إلى 


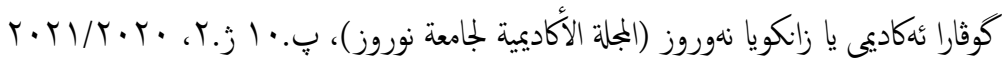

"عادة ما يتم إعطاء قيمة 1 بيتكوين (BTC) بالدولار الأمريكي وقد تغيرت هذه

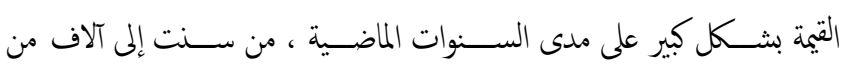
الدولارات وبسـبب هذا فإن النظر في مبالغ الصرسف مباشرة في BTC قد لا تكون مُثلة لقيمها الحقيقية ، وبالتالي قُنا بتحويل BTC إلى USD باسـتخدام

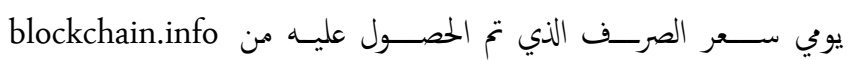
(Erdugan, 2012, p. 8) هي أصسول مضـاربة، في حين يعتبر الاتحاد الأوروبي أن Bitcoin وغيرها عملة، من ناحية أخرى تعتبر اليابان أن العملات المشـفرة هي شـكل من أشـكال الدفع المسبق الماثل للائمان و بطاقات الهدايا (4odricks,2018: ) .

\section{The Concept of Cryptocurrencies مفهوم العملات المشفرة 2.1.2} تشير المفظة الرقية إلى نظام إلكتروني يسمح للفرد بإجراء معاملات إلكترونية وتشـمل شراء البنود على الإنترنت باسـتخدام الهاتف الذكي او من جماز كبيوتر أو لشراء شيء ما في متجر.كما يككن ربط الحسـاب المصرسفي الفردي بالمفظة الرقية، وتمرير وثائق التفويض إلى محطة التاجر لاسلكي عبر الاتصالات الميدانية القريبة على نو متزايد، ويتم إجراء محافظ رقية ليس فقط للمعاملات المالية الأساسية ويكن تستخدم لمصادقة آوراق اعتاد صاحها (أبو صلاح، 2018: 16) لمعرفة ماهية العملات المشـفرة لابد من توضــيح مفهوم العملات المشــفرة لغة

$$
\text { واصطلاحاً ومن ثم التطرق المى الجانب النظري فيها : }
$$

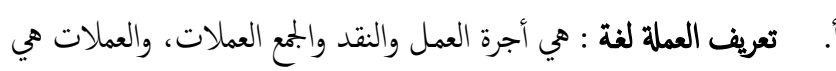
نقد يتعامل به الناس (لفيروزابادي،2005: 1350)."والافتزاضــية :اسم مؤنث منسوب إلى افتراض ، وهو مصدر صناعي من افتزض افتراضا، أي

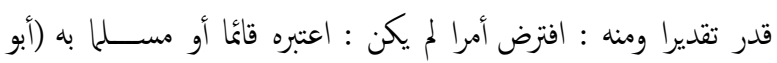
حسين،2019:113) - (20) - (2) - (2) ب. تعريف العملة اصـــلاحاً : "تعرف العملة بأهها وســيلة التعامل المال بين الناس (عساف،2019:24) . تعريف العملات المثـفرة :"العملات الرقية المشـفرة هي وسـيلة للتبادل النقدي حيث يتم تخزين الثيمة ونقلها إلكترونيًا (Thornton,2016:8) . "هي عملة رقية تم إنشــاؤها في عام 2009 ، يقوم بتقديم آلية دفع دون أي رقابة مركزية ( أي من قبل المؤسســة أو الحكومات أو البنوك) ، حيث يتم تسـجيل جميع معاملات Bitcoin بالفعل في blockchain ، وتكون

\section{4anardag 2019 (Ya.2.1 عنوان الدراسة}

\section{Cryptocurrency Investment Decisions and Behavioral Bias}

Effect

هدفت هذه الدراسة إلى دراسة محددات كليها ملكية العملات المشفرة وعلى استعداد للاستثمار في العملات البديلة ، باستخدام الأشخاص المعلومات الديموغرافية وميل التهيزات السلوكية الثقة المفرطة ، والبحث عن المخاطر ، وتنور الغموض ، وتجنب الخسارة، تم أستخدام نموذجين نموذج الانحدار اللوجستي (OLS) والعنوذج انحدار خطي من خلال (ORM) أستطلاع على الأنتزيت بطرح (34 سؤال) عبر منصة مع (304 فرد) لفترة 2019/4/22 الم 2019/5/7 ، بينت هذه الدراسة أن المعرفة المالية تزيد من ملكية العملة المشفرة ،وأن مستوى التعليم المنخفض يقلل من ملكية العملة

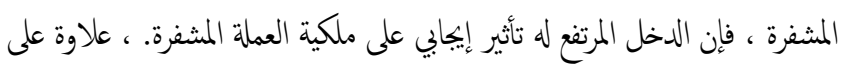
ذلك ، فإن وضع الدخل المرتغع له قيمة تأثير إيجابي على عدد ملكية العملات الرقية

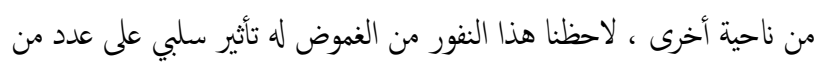

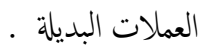
2. المحور الثاني : الأطار النظري للبحث 1.2 مدخل نظري حول ماهية العملات المشفرة والعوامل المؤثرة فها A Theoretical Introduction about What Cryptocurrencies are and The Factors Affecting Them

The Origins of Cryptocurrencies 1.1.2 نشأة العملات المشفرة "بدأ التفكير في طرح عملة وهمية (افتراضـية) عام 2007 من قبل مبرمج مجهول الهوية يعرف باسم Natoshi Nakamoto ، قام Nakamoto في عام 2008 بنشر- ورقة عمل بعنوان Bitcoin نظام عملة الإلكترونية " الندّ للندّ "في موقع خاص بالتشفير الاكتروني ييين فيه طريقة عمل عملة Bit وحايتها من التزييف والإنفاق المزدوج ووصـفها بأهها نظام نقدي الكتروني يعتمد في التعاملات المالية المباثرة بين طرفين دون وجود وسيط ثالث ، اصل هذه الفكرة كان محاولة من Nakamoto للنق عملة غير خاضـعة للرقابة والهدف من ذلك لمواكبة التغيرات المتسارعة في عالم الاعمال خاصة على الشبكة الافتراضية وترير الاقتصاد العالمي لتلافي مشـاكل النظام النقدي التقليدي من بجة من بجة اخرى (المود،،2019: 


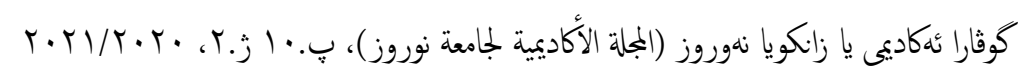

إنّ العملات المشـفرة حلقة في سـسـة تطور العصر- الرقي، اي هي مجال التعاملات الكلكترونية النقدية، فهي تسعى لكسـب سـات العملات الورقية ووظينها وســات النقود الالكترونية لإقامة نظام نقدي عالمي جديد، تحقيقها لليمقراطية نقدية مصسـرها الثـعوب وليس القوى المالية العالمية والسلطات المكومية الوطنية.

اعتمدت العملات المشفرة على خصائص الرياضيات وتعقّد نظاهما بشكل لم يسـبق له مثيل ، قد يكمن وراء بعذ اقتصـادي عالمي، تجديد ظهور الرأسمالية بظهر جديد يكون أشد بشاعة في تحطيم اقتصاديات الدول لتركيز الثروة بيد قوة متحكمة واحدة، تنفرد بالقوة الاقتصـادية العالمية، ظاهره الانقلاب على النظام الرأســالي على النظام الاقتصــادي العـالمي واقتصاديات الدول الناشئة،. عدم خضوع العملات المشفرة للرقابة ما يصعب تتبعها ولايسيطر عليها من قبل الســلطات المالية المكومية، مما يضــف نظامها الاقتصـادي المتمثل بالأبتعاد عن التعامل بعملاتها الرسمية، وإضـــعاف مواردها كما في التهرب

$$
\text { الضريبي والخروج من سلطة الحكومات الوطنية . }
$$

هجمت العملات المشفرة هجمة مضادة على أنظمة تتبع تمويل الإرهاب وغسل

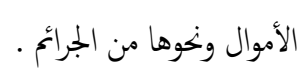

العملات المشفرة قد تجد قبولا من الدول الاقتصادية الدولية والتي تسعى المى موابحة العقوبات وتنفذ هذه العقوبات المؤسسات المالية العالمية الكبرى

$$
\text { حيث تقوم بتبادل النقد والتحويلات النقدية . }
$$

تجد العملات المشــفرة قبول من الدول في موابحة أزمات التضــخم لدعها

$$
\text { للعملة الوطنية. }
$$

\section{Properties of the Cryptocurrency 4.1.2 خصائص العملات المشفرة}

تتميز العملات المشفرة بمجموعة من خصائص العملة المشفرة (محم، 2016 : 28) (الباحوث، 2017: 31) (عامر، 209: 277) (رسلان، 2019: 461) :

عملة رقية افتراضية وهية ومشفّرة غبر ملموسة وليس لها وجود فيزيائي،لا وجود لها في أيدي الناس بشـكل حقيقي، أي لايمكن تتبع عمليات البيع

$$
\text { والثراء التي تتم بها حول العالم. }
$$

مشتركة مع دفتر الأستاذ العام، تم تنظمه بترتيب زمني داخل سلسلة الكتل ويؤمن بفضل تشفير قوي باستخدام برامج المستخدم النهائي المسماة عملاء

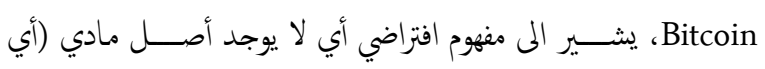
ملموس) ، توجد العملات المشفرة في أشكال رقية وإلكترونية في الإنتزنت يتم تسجيل العملات في حساب الحاسوب .من (World Wide Web) أثـهر الأمثلة للحسـاب الرقي هو مصــلح المحفظة المسـتخدم لوصـف الحساب الذي يكنن تحديده بشكل فريد لمالكي البيتكوين وسهل الأستخدام بسهولة (Erdugan,2012:2) ."ويكن توضيح مفهوم العملات المشفرة من خلال النعاريف التي وردت بجقها والتي تعددت بتعدد وبجات نظر الباحثين والجهات التي أولته اهتماما نذكر منها : العملة المشفرة هي أموال رقية في نظام دفع إلكتروني، بواسطة شبكة لا مركزية يتم التحقق من صحة المدفوعات فيها من مستخدي النظام وبروتوكلات التشفير بدلاً

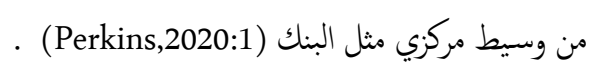
العمة المشفرة هي نوع من أنواع الأصول الرقية المصمة للعمل كوسيلة التبادل باستخدام التشفير لتأمين المعاملات ، للسيطرة على إنشاء، التحقق من نقل الأصول وحدات قيمة إضافية (Härdle et al,2019:3). أنها شكل من أشكال المال موجودة فقط على الإنترنت وتسىى بالعملة المشفرة، عن

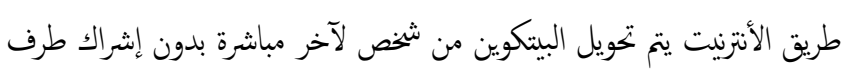

(Ghimire \& Selvaraj,2018:2). ثالث مركزي مثل نظام المصارف عرفت العملات المشفرة بأهها تثثيلات رقية للقيمة وفي نس الوقت ليست صادرة

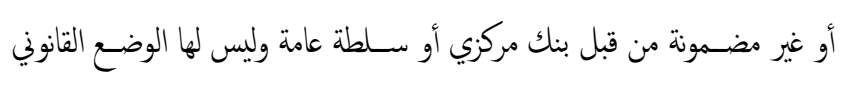
للعملة المشفرة أو المال (Berentsen \&Schär,2018:5). مما تقدم من تعاريف واستناداً لما طرحته الأدبيات الفكرية للعملات المشفرة هي عملات أفتراضية لامركزية لاتدعها كيان قانوني غير ملموس وغير متجانس تتم من بين طرفين عن طريق الأنتزنت بدون وسيط مثل البنك .

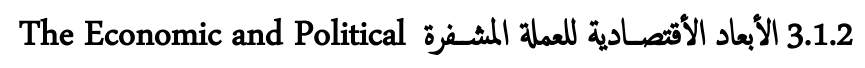
Dimensions of the Cryptocurrency للعملات المشــفرة بعض الأبعاد الأقتصــادية حســبـ رأي كل من (لمهداوي والعيساوي، 2019: 529) نذكر منها الآتي : 
الرسوم المنخفظة: لايوجد مصاريف للتحويل والنق لعدم وجودوسيط بين طرفين لنقل المال كالتي تتقاضاها شركات بطاقات الائمان والبنوك عادة . السرية والخصـوصية: نظراً لطبيعتها الخاصـة لايمكن مراقبة عمليات البيع والثرـاء، كما أنها تقلل سـيطرة الحكومات والبنوك على العملة حيث يمنن نقلها في اي مكان واي وقت وبخصــوصـية تامة بدون ان تمر على بنك أو هيئة رقابية. العالمية: يككن تحويل العملة الرقية من بلد المى أخر بسهولة بدون تعقيدات ولا يمكن أن تتعرض للمصادرة أو للتجميد والأكثر أهمية تكون بدون كلفة، فهي لاتخضع للحدود الجغرافية. - افي السـلامة من المخاطر: فلا يككن لأي دولة حظرها أو الحجز على تعاملاتها وتحويلاتها أو مصادرتها أو تجميدها لأنها لا تخضع لسيطرة ابتداء . سهولة الدف: تسمح العملات المشفرة لمستخدميها أن يتحكوا في أموالهم بشـكل كامل، حيث من الممكن إرسـال واسـتقبال أي مبلغ من الأموال لحظيَّا من والى أي مكان في العالم وفي أي وقت،لا قيود ولا حدود

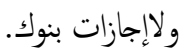

الشـفافية : يقوم البرنامج بخزن أي عملية تم القيام بها التي يمنكها صـاحب المحفظة يككن لأي شخص معرفة عدد المعاملات التي تمت وعدد وحدات البتكوين، ولايسـتطيع احد معرفة هوية مالك المفظة، يككن لمستـتخدميها تشــنيل وممارســة الأمان لماية اموالمم، حيث أن مزودي الخدمة يقوموا بتوفير درجة عالية من الأمان ضد السرقة والتأمين ضد الخسارة، (البرتوكل) المستخدمة واحدة من اكبر مشاريع الحوسبة الموزعة في العالم مما يجعل من

$$
\text { الصعب تزويرها أو اعادة استنساخها. }
$$

The Risk of cryptocurrencies 6.1.2 ان العملة المشفرة كغيرها من وسائل الدفع الحديثة هي نتاج التطورات الحاصلة في الحياة البشرسية و البيئة التكنولوجية لذلك فهي معرضــة للخطر نتيجة تدخل اشخاص او اطراف خارجية تندخل بالصفقات من جهة ، كذلك يكن ان تنجم مخاطر عن طبيعة هذه العملة كخدمات مالية تعتمد على التكنولوجيا الحديثة و فيا يلي بعض من هـــه المخاطر (الجزراوي، 2011: 46-47) Bohme et al,2015:216 (الباحوث، 2017: 36) (رسلان، 2019: 462) :
عملة غير نظامية، بمعنى أنها غير مدعومة من أي بجة رسمية ، منظمة دولية، مؤسسية، إمكانية إصدارها من أي شخص في العالم يتقن استخدام علم البرجيات والحاسوب والخوارزميات الرياضية، يناح لجميع المتعاملين بها إمكانية تعدينها بحسب إمكاناتم التقنية والفنية تستخدم من خلال الإنترنت فقط، وفي نطاق المؤسسات والشركات والهيئات والمواقع الإلكترونية التي

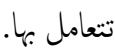

عملة سـهلة الحمل وثنائية الاطراف دون حاجة لتوسـيط البنك وليسـت متجانسة. بواسطة أبهزة صرافة آلية خاصة أو مواقع متخصصة يكن تبادلها بالعملات الورقية الرسمية مثل الجنيه والدولار واليورو ، بعمليات مشفرة عبر الإنتزنت. ترتغع وتنخضض بشـكل جنوني في فتزة وجيزة بسـبب التذبذب الكبير في أسـعار هذه العملات، ، فقد يتحقق لم ريخ عالية أو خسـارة كيرة غير متوقعة في عشـية وضحاها ومن غير مقدمات ومن غير وجود أسـباب منطقية أو تحليل اقتصادي بجيث يكون المالكون في مخاطرة كيرة . عدم قدرة دولة أي السلطات النقدية في مراقبة العمليات التجارية التي تتم بواسطنها والتحكم في سعرها أو عرضها.

نظراً لطبيعها اللامركزية واعتمادها على التقنية وتجاوبها مع مســتجداتها وتطورتها، تعتبر آلية ملائمة للشراء والبيع بطريقة سلسة وسريعة. التعامل بالعملات المشـفرة يكون على شـبكة الإنترنت فقط، فلا يكن الحصول عليها عن طريقة مراجعة البنوك أو أية مؤسسة مالية أخرى .

\section{The Advantages of Cryptocurrencies 5.1.2 مزايا العملات المشفرة} تمتع العملات المشــفرة بالعديد من المزايا ونذكر منها ما يلي (عبدالحفيظ،2010: 42) (البـجـاري وامــ،2018: 16) (شـــافي، 2007: 88) (Carr \&)

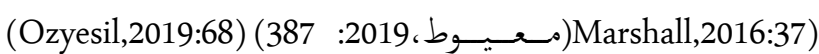
: (Ozyesil, (كيد، 2019: (International Bank,2018:44) 2019 , p. 68) السر-عة: عملية التحويل والدفع الملالي تكون سريعة حيث تتم فور ضـغط

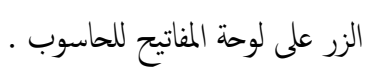




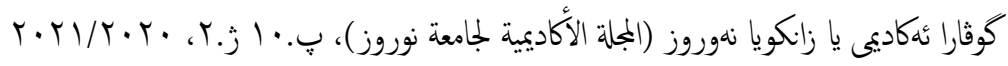

أهم أنواعها (Bitcoin) في نهاية 2016، ثم أزدادت خلال اقل من شهرين (حتى 16 شـــر مارس 2021) الى (8800) عملة مشـفرة، أي أزدادت عدد العملات المشفرة الجديدة بواقع اكثر من (1500) عملة، حسب أستقصاء الباحثة من موقع coindataflow.com : تعتبر أول عملة رقية مشفرة ويرمز Bitcoin على الشبكة العنكبوتية ولكنها ليست الوحيدة، وقد تم كثـف النقاب عنها في ورقة بحثية نشرت في عام 2008 من شخص مجهول الهوية يدعى Nakamoto يتخفون وراء هذا الوسم المستعار ، وقد تم البدء في تداول عملة البيتكوين في عام 2009 (صلاح، 2015: 22) . BTC عبارة عن عنوان رقي مربوط بحفظة إلكترونية، وكل Bitcoin

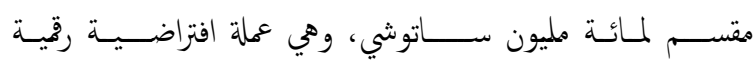
(cryptocurrency) لها، تعمل بشبكة الند للند (per-to-per) بين المستخدمين مباشرة دون طرف ثالث من خلال استخدام التشفير و يتم التحقق من المعاملات عن طريق ما يسمى بسلسلة الكتل المغلقة (الناطور ، 2019: 324) . BTC لا تصدر عن بنك مركزي أو سلطة نقدية مما يعني عدم وجود أي سلي جحة رقابية أو اشرافية علهيا، تعمد بشكل أساسي على مبادئ التشفير ليس لها وجود مادي سـواء معدني أو ورقي ويتم استخدامها فقط عبر شـبكة الإنتزنت لتسـوية المشتريات أو تخويل العملات، وذلك عن طريق انتقال الكود مباشرة من محفظة المشـتري لمفظة البائع، وهي تشــهـ الحسـاب البنكي فهو آمن وسري و شخصي (حامد،2014:15) . Litecoin

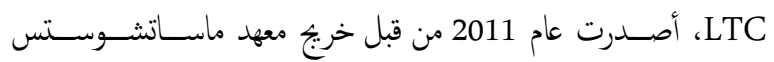
للنكنولوجيا، "تثــارلي لي" كان يعمل سـابقاً همندسـاً لدى شركة google قدمت عاسها باعتبارها العملة الفضية مقارنة بعملة BTC الذهبية، اذا كان البتكوين هو الذهب فإن اللايتكوين هو الفضة، وتتميز عن البتكوين بأن عملة التنقيب أسهل ، حسب رأي الختصين بأن التعامل بعملة LTC أسرع من عملة BTC لكن من حيث الشهرة والقيمة السوقية تبقى عملة BTC في الصدارة (عامر، 2019: 273) .
اعتداءات اجرامية كالسرقة الاحتيالية للمعلومات المالية، واعتداءات

$$
\text { من قبل مقتحمين كالعبث بالمواقع الاككترونية وتعطيلها. }
$$

2.6 سوء استخدام النقود من قبل مالكه سواء كان بشكل مقصود

او غير مقصـود، من الممكن ان يزداد التهديد بسـوء الأسـتعهال في

حال لم تقم الجهات المصـــرة لها الدقة بأســتخدام العملة باعطاء المستهاك معلومات وافية حول المخاطر الامنية لهذه العملات.

أستسخراج نسخ مزيفة عن العملة المشفرة وذلك بعد الحصـول على تفاصيل العملة الاصلية بطريقة غير مشروعة، عدم خضوع العملات المشـفرة لسـياسـة البنك المركزي وعدم وجود اسـاس قانوني مثل

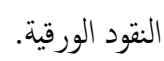

تذبذب الكيبر للأسـعار على عكس النقود القانونية التي يتمع بدرجة

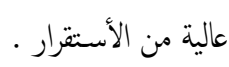

الافتقار للحاية القانونية، فطبيعة هذ العملة هي أنها لا تصدر عن أي مؤسســـة رسمية مثل البنك المركزي ، وعدم وجود بهة أشرافية يجعل هذ العملة تثتقر للقيادة المركزية القادرة على اتخاذ القرارات الحاسمة والسريعة، كما أنها لا تخضع للرقابة المالية المختصة ."وهناك مخاطر اخرى مثل : مخاطر الطرف المقابل ، مخاطر المعاملات ، خخاطر التشـيل ، المخاطر المتعلقة بالخصـوصـية ، والخخاطر القانونية والتنظيمية. يواجه أي مستخدم يمتلك العملة المشـفرة مخاطر السـوق من خلال التقلبات في سعر الصرف بين العملات المشفرة والعملات الرسمية ، التهرب الضركيبي مثل تهريب الأموال المى الخارج أو تحقيق خسـائر وهيمنة من خلال عمليات الشراء، خخالفة القوانين مما تسهـل

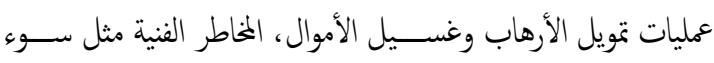
أسـتخدام اداء شـبكات الأتصال او أختيار نظام لايواكب التطورات المتلاحقة في تقنية المعلومات، عدم وجود ججمة الانرافية مما يجعلها تثتقر للقيادة المركزية القادرة على أتخاذ القرارات الحاسمة والسريعة".

\section{The Most Popular and العملات المشفرة الأكثر شهرة وتداولاًا}

Traded Cryptocurrencies "إزدادت بشكل تدييمي عدد العملات المشفرة بعد الطفرة التي حدثت في أسعار 


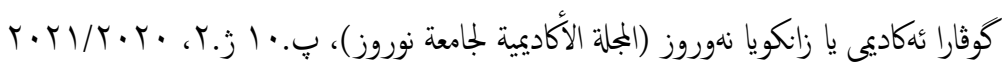

لمؤسسات المالية بتسوية المدفوعات عبر المدود بشكل أسرع وأرخص ما يككنه اســتخدام شــبكات الدفع العالمية الموجودة اليوم ، والتي يكن أن تكون بطيئة وتشمل وسطاء متعددين (أي البنوك) ـ مكن لـ XRP معالجة أكثر من 1500 معاملة في الثانية ، لايعتمد XRP على PoW أو آلية PoS للتحقق المعاملات، لكنها تستخدم بروتوكول التوافق الخاص بها Snyers ) \& Robby ,2018: 39) Ethereum العقود الذكة، بطريقة تحاكي إبرام العقود التقليدية، لكنها تتطلب شروطاً ومتطلبات لتنفيذها دون الحاجة إلى سلطة أو بجة معينة تتحكم في عملياتها. اقتحما المبرمج الروسي Vitalik Buterin عام 2013، وفي عام 2014 عملت شركة سويسرية على مشروع عملة والبيتكوين إلى أن تم إطلاقها بشـكل رسمي عام 2015 تقدّر قيمتها السـوقية بأكثر من (116) مليار دولار، وقد تعرضت المنصة لعمليات اختراق وسرقة نحو 50 مليون دولار في 2016 ، وبعد هذا لهجوم تم تقسيم الإثريوم إلى إثريوم ETH وإثريوم كلاسيك ETH ، و و هي ثاني أعلى عملة رقمية قيمة بعد BTC (الجميلي، 2019: 90) ـ الميزة الرئيسية ETH على BTC هو أن الأول يوفر الدي لأنواع ختلفة من التطبيقات اللامركزية، زادت تكلفة منصــة ETH أكثر من الضــف ETH , BTC تنافس في سـوق العملات الرقية ككانت حداثة ETH امتدادًا لجمموعة من التعليات في Java كامل المواصفات لغة برجة الفسكريبت يبلغ الرسملة الإجالية لسوق العملات الرقية الآن 202.5 مليار دولار .أصبحت ETH أرضية انظلاق لمشاريع مختلفة ونتيجة لذلك ظهرت جاذبية الاستثفار (ICO) في شكل مبيعات للمستتمرين تلقت من قبل واحد أو الإصـــار المعجل لعدد ثابت من وحدات العملة المثــفرة

.(Parshina et al,2019: 245) Evan Duffield في عام 2014 ، وتزمز :"أسسـها Bitcoin Cash BHC ، وتسمى بالعملة الماكنة BTC لديها المزيد من الخصوصية مثل لا يككن تعقب المعاملات، كما لها وظائف موزعة على شبكة رمز رئيسية، وجدت عملة BHC في 1 أغسطس 2017 أصببحت هذه العملة شـائعة بين العديد من الناس حول العالم. مما يجعلها لا تزال عملة جديدة نسـياً. بينما تظهر العملات المشفرة الجديدة في كل فترة وفترة، بعضها متمز بشكل كير
عملة LTC هي عملة مشفرة لامركزية P2P مفتوحة المصدرر ، ويستند إلى مايعرف باسم خوارزمية Scrypt PoW ، التي تســخدم BTC ، خوارزمية SHA-256 PoW الأصـلية وبصر-ف النظر من حقيقة أنه يستخدم خوارزمية ختنلفة ، فهو خختلف عن BTC بطريقتين : O وينج عن اسـتخدام خوارزمية Scrypt PoW ، تقدم LTC O على BTC في المعاملة من حيث السر_عة الوقت اللازم لإنشــــاء سـلسـة الكتل المغلقة على BTC حوالي عشرة دقائق، في حين أن متوسـط وقت إنشـاء ســسـلة الكتل المغلقة على LTC هو 2.5

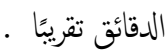
O ميلغ الحد الأقصى-لإمدادات LTC (84 مليون ) عملة ، وهو أعلى بكثير من 21 مليون حـد العرض BTC ، تعمل مTC على سلسلة الكتل مغتوح بدون تصرئ تمامًا مثل \& BTC (Robby .Snyers,2018:41) من أهم وأثشهر العملات المشفرة الحالية على مستوى العالم : Ripple ويرمز (XRP)، حيث تحتل المرتبة الثالثة عالمياً من حيث السـيولة، حيث تتدفق الأموال بحرية كاملة، عبارة عن منصــة دف رقمية مفتوحة المصدر ومزودة بتقنية P2P تتيح تقريبًا تحويلات فورية للعملة بغض النظر عن شـــلها (مثل الدولار الأمريكي والين والبيتكوين ...) أنشــــت عام 2013 بإنتاجما شركة تسـى ( أوبن كوين)،وقد تم إنشـاء شـبكة لعملة لتسـمح بتناقل أي شكل من أشـكال العملات بسلاسـة ويسروسهولة، سواءكان الدولار أو الجنيه او الين أو البيتكوين ، حسب رأي الاقتصاديين فإن الوقت هو العامل الوحيد في ارتفاع سعرها في المستقبل القريب، ومن المحتمل أن تحل محل البنوك في المسـتقبل وذلك لسرـعة التحويلات المالية وسهولتها (شعبان وآخرون، 2020: 24) أطلقت في عام 2012 من قبل الشركة الخاصة(Inc ، Ripple (Labs)، المسؤولة عن مزيد من تطوير بروتوكول Ripple ، هي أول شركة تحصل على "BitLicense" لحالة استخدام مؤسسي_ للأصول الرقية من إدارة الخدمات المالية في نيويورك، كما حصـلت على الدع من عدد من اللاعبين America Merill الكبار في صــناعة الخدمات المالية ، مثل بنك

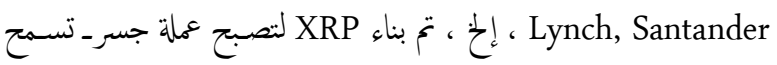




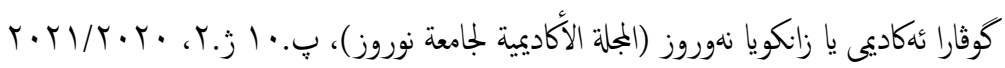

خلال تنفيذ نظام التعدين حيث الناس في الشـبكة، تسمى عال المنابم أو العقد

. (Krishnan et al,2015:115)

تعدين العملة المشـفرة هو عملية إنشـاء عملات جديدة عبر التحقق من المعاملات في شسبكة المشفرة، يتم استخدام أبهزة تعدين العملات المشفرة المخصصة لهذا الغرض، تستخدم لحل مشكلة رياضية في المقابل يحصل عال المنابم على 25 عملة بيتكوين، يتم إنثـــاء كتلة جديدة كل 10 دقائق تقريبًا مما يؤدي إلى إجلالي 1.3 مليون بيتكوين أو 845 مليون دولار سنويًا في التقييم الحلالي، تشكفة التحقق من المعاملات هي النكلة الرأسالية لشراء عال المنابم واستهلاك الطاقة للتشغيل الأجهزة بالإضــافة إلى النفقات التشــيلية للحفاظ على تثـــيل العملية

.(Kampl,2014:3)

يتم تأمين شبكة العملة المشفرة بواسطة أفراد يدعون عمال المنابم .يكن لأي بجاز في شـبكة Bitcoin أن يعمل كنجم .اسـتخدم المسـتخدمون عدة أنواع من الأجزة بمرور الوقت للتنقيب كتل بيتكوين .تعدين وحدة المعالجة المركزية ، تعدين وحدة معالجة الرســومات ، مصــفوفة البوابات القابلة للبرجمة الميدانية و الدائرة المتكاملة الخاصـة بالتطبيق هي أبهزة شـائعة الاسـتحدام لبيتكوين التعدين .كل تعدين الأجهزة يجب أن يتعامل مع الربح المنخض ، الحرارة الزائدة وارتفاع تكلفة الكهرباء (Ghimire \& Selvaraj,2018:4) .

"الغرض الرئيسي_ من التعدين هو توليد وإطلاق عملات معدنية في اقتصــاد العملات .عندما تأخذ المعاملة مكان وصدقت عليه عال المنابم جمع هذه المعاملات و تضـمينها في الكتلة التي يقومون بحلها حاليًا، يجب حل الكتلة قبل بثها ووضعها في سلسلة كتلة، هذه الآلية تنع عال المنابم من شراء العملات بسهولة وبالتالي يحافظ على عدالة النظام يتضـمن حل الكتلة الألغاز الرياضية التي يصعب فتحها وكسرها شريطة أن يكون هناك ولدت بعض القيود على الإخراج، فقط على حل اللغز الرياضي هو واحد يسمح بإضافة كنلة إلى يتم إعطاء دفتر الأسـتاذ ومكافأة العملات في المقابل وبالتالي التعدين تتلخص في نهاية المطاف في مسابقة رياضية الألغاز لحل مكافأة العملات المعدنية (Chiu \& Koeppl,2018:7) . يتم تعدين عملة التشـفير من خلال الآلات المصـمة خصـيصـا تسـى الآلات التعدين، يبدأ تاريخ الآلات التعدين من وحدة المعالجة المركزية CPU إلى الوقت الحالي ASICs تستخدم على نطاق واسع، لصعوبة النعدين أدى إلى تطور آلات جديدة بكفاءة أعلى من الآلات المصسمة سـابقا، يتم تحديد آلة التعدين ربحينها عن
مثل عملة BTC ، وبعض الآخر عبارة عن نســخ مقلدة إلى حد ما، و الفرق الحـاسم في عملة BHC هو أن أصـــولها تـأتي من عملة البيتكوين الأصلية (Ibrahim \& Almansour,2020:3). Pascal Coin’s وتداولها أسهرل Pascal Coin's بكثير من BHC ، وأرقام الحسابات التي يحصل عليها من اشتراها أرقام قليلة لا تزيد على عشرة أرقام، فهي مبنية على لغة البرجة الشهيرة باسكلال تعالج بعض سلبياتBHC وتعمل بشكل مشابه للبنوك، تعتبر Pascal هي العملة الوحيدة التي بامكانها القيام بمائة معاملة في الثانية Coin’s الواحدة ، كما أن Pascal Coin's تعمد بشكل كامل على سلسلة الكنل المغلقة حيث هناك تكنولوجيا جديدة تسـمى SAFE BOX تعالج سلبيات سلسـلة الكتل إذ تحتفل سلسـلة الكتل بجميع المعاملات دون حذف، مما يسبب الكثير من المشاكل أهها ازدياد حجم سجل التعاملات مع مرور الوقت بشكل لايمكن السيطرة عليه. باسكال كوين تحتفل فقط بأخر (100 كتلة) وغير ذلك يكن حذفه بدون أي مشـكة على أمان الشـبكة

$$
\text { (الجميلي، 2019: 178) (179) }
$$

\section{Factors affecting 2.2}

cryptocurrencies

"سوق العملات المشفرة متقلب للغاية، تصل التقلبات الاسعار في بعض الححيان الى 20\% أرتفاعاً وهبوطاً خلال اليوم الواحد، اسـباب التغير غير معروفة بشكل دقيق في بعض الأحيان، اختلف الباحثين في تحديد العوامل او المتغيرات التي تؤثر على قيمة العملات المشفرة في الاسواق كما الآتي (Vogel,2019:10)

\section{Internal Factors العوامل الداخلية 1.2.2} Difficulty Of Mining 1.1.2.2 التعدين هي عملية متكمالة من حيث التوليد، بطريقة تجعل جميع المعاملات مصــوعة من بداية العملة المســجلة و يتم الاحتفاظ بها في دفتر الأســتاذ العام ويسمى (سلسلة الكتل) وهي قائمة من الكتل التي تم إنشاؤها من البداية، يضمن نشر- ونقل مســتقر وآمن للعملة من البائع إلى المســتفيد على عكس العملات الورقية ، صممت من أجل نقل العملات من حفظة إلى أخرى، وترصد المعاملات تتطلب بنية تحتية ضخمة للعمل، تتغلب العملات المشــفرة على هذه الحاجة من 


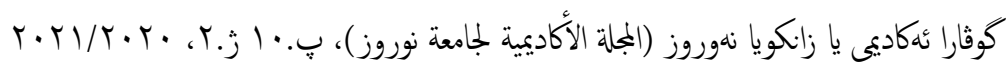

ASICs

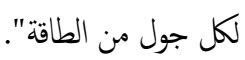

Economic Variables 2.1.2.2

تعتبر متغيرات الأقتصادية الجمموعة الدافعة الرئيسية التي لها تأثير مباشر على سعر

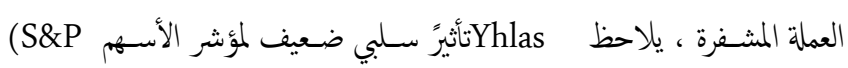
(500على سـعر العملة المثــفرة ولا ســيما البيتكوين ـ أن أي زيادة في مؤشر الأسهـم S\&P 500 يتسـب في الخفاض أسـعار البيتكوين على المدى القصـير حيث توجد علاقة عكسية موثقة في المدى الطويل، وهذا يؤكد العلاقة السلبية بين أسعار العملات المشـفرة وأسـعار سـوق الأسهم على المدى القصير والمدى لئ.

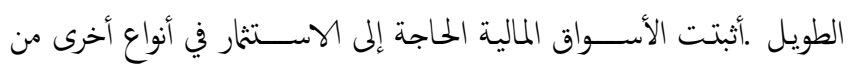

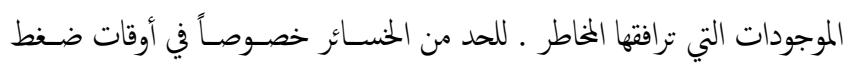
السـوق لابد من توفر هذه الموجودات لدى المســتمثرين (8hlas,2018:7) . (Jeong,2018: 17-19)

\section{Supply and demand 1.2.1.2.2 العرض والطلب}

أذا كان لدى العملة المشــفرة عرض عالٍ مع قلة في الطلب من جانب المسـتمثرين فسـوف تنخفض قيمة العملة المشـفرة، وبالعكس إذا كان عرض العملة المشــفرة محدود وكان الطلب عالٍ فستزيد قيمة العملة المشـفرة . يرتبط هذا بعنصر الندرة الذي يدفع الأسعار ويمثل احد العوامل التي أدت المى أرتفاع اسعار Bit الى اعلى مسـتوياته، حيث يتوقف الإمداد في Bit عند 21 مليون Bit وهو منخفض

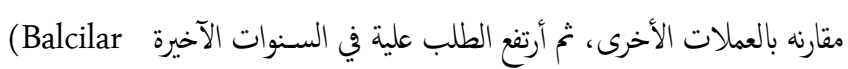
. et al,2017: 19) تعتمد الأسعار على العرض والطلب ، لذا فإنه يتم تحديد السعر عنده يككن تبادل العملات المشفرة بعملة أخرى يككن أن تتقلب على نطاق واسع .فمثلا، بلغت قيمة 7000 Bitcoin دولار في اليوم التالي، يعتبر بعض الاقتصاديبن ترتبط تكلة إنتاج العملة المشفرة ارتباطًا مباثرًا لســعر الســوق بمجرد أن يمناك شخص ما عملة مشــفرة حيث تستهاك كية كيرة من الكهرباء ، يككن للناس استخداهما العملات المشفرة لشراء السلع والخدمات عبر الإنتزنت أو تخزيها ( Janicki,2019:8 ) :

Inflation 2.2.1.2.2 هو انخفاض القوة الشرائية للعملة ، تتعرض معظم العملات المشفرة للتضخم عندما
طريق التكلفة والأداء، التصــميم والتنفيذه أمر بالخ الأهميـة، الآلات الخختلفـة

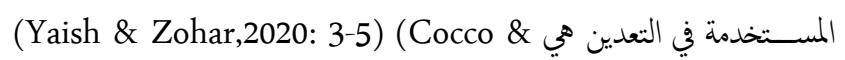
.Marchesi,2016:3-4) (Krishnan et al,2015:116-117)

CPU 1.1.1.2.2 وحدة المعالجة المركزية

في بدايات التعدين تم اســتخدام وحدة المعالجة المركزية للتعدين عملات معدنية بشكل فعال ، جهاز حاسوب شخصي- مثبت عليه برنامج تعدين كان كافياً للتعامل مع عملية التعدين بمعدلات تجزئة أقل أو تســاوي 10ميجا هرتز / ثانية ولكن بسبب زيادة مسـتمرة في صسعوبة التعدين واسـتخدام وحدات المعالجة المركزية أصبحت آلة النعدين ذات صـاة بالآلات المتطورة مع معدلات تجزئة أعلى برنامج تعدين شائع لوحدة المعالجة المركزية هو cpuminer . GPU 2.1.1.2.2 وحدة معالجة الرسومات نظرًا لأن قوة تعدين وحدة المعالجة المركزية (CPU) لا تلبي النمو المطالب ، يتم اســتخدام وحدة المعالجة المركزية مع بطاقات الرســومات لتعدين القطع النقدية. تحتوي بطاقات الرســوم البيانية على وحدات معالجة الرســـومية (GPU) التي تستخدم لحل الحسابات الرياضية العالية الوظائف والمضلعات المعقدة المستخدمة في الألعاب وبالتالي كان ينظرالى GPUs كبديل موثوق به لتعدين CPU . FPGA 3.1.1.2.2 مصفوفة البوابات القابلة للبرجة الميدانية في يونيو 2011 بيعت أول FPGA Bitcoin مفتوح المصـدر تطبيقات النعدين مع الزيادة المسيتمرة في التكلة التعدين مقابل العملات المعدنية المكتسبة، أثر ذلك بك

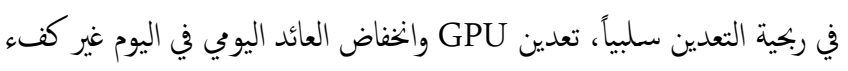
لمنجم او أكثر مع ارتفاع تكلفة التعدين .وبهذا ظهرت الحاجة الفورية والطارئة للألات التي يمكن أن تجعل التعدين مربح لعال المنابم من اجل مواصلة التعدين".

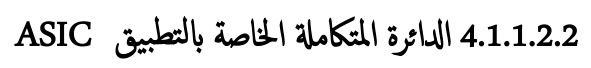
اصبح من الصعب الحصـول على عملات النعدين مع مرور الوقت وذلك لوجود الآلات المطورة المتاحة بأرخص الأسـعار جعلت المنافسـة ضخمة بين عال المنابم لتحقيق المزيد من المكلســب من خلال التعدين . FPGAs مصـممة لغرض

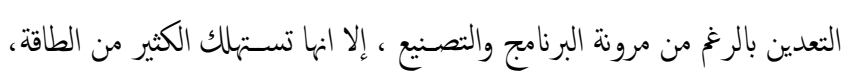
تستخدم ASICs للتعدين على نطاق واسع، تم تصميم الدوائر على وجه التحديد لحساب النجزئة بأسرع وقت مككن مع استهلاك أقل قدر مككن من الطاقة .أفضل 


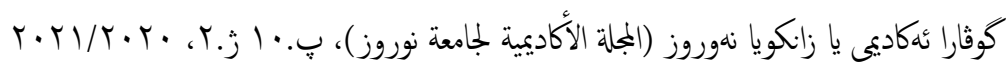

بمدخراتهم في أصـول المضـاربة خلال فترة عدم الاسـتقرار ، في حين يعتقد البعض الآخر أن هذا الحدث ســيؤدي إلى اسـتخدام عدد كبير من الأشـخاص للعملة المشـفرة كأصـل مخزن القيمة. من خلال مكاففة عدم الاسـتقرار ، خفض مجلس

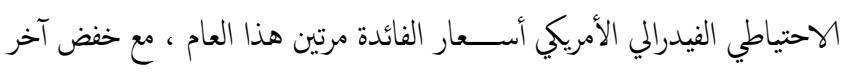

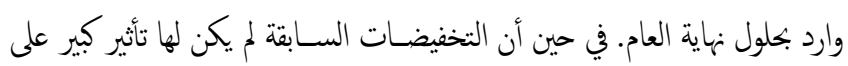
سوق التشفير ، إلا أن زيادة الخوف من أن مجلس الاحتياطي الفيدرالي غير قادر على وضع حد للأزمة قد يؤدي إلى وضع الأشخاص لمدخراته في سوق إلكتروني لأصول ذات قيمة مثل العملة المشفرة (Jankov,2017:3) .

Technology النكولوجيا 4.2.1.2.2 "سـهولة شراء العملة المشـفرة مع اهتخام الزائد بالوسـائط ادى الى تدفق مشترين جدد اقل معرفة مالية وهناك جهود نشطة جارية لجعل السوق أقل غموضًا، حيث بدأت الجامعات وما حولها في العالم في تقيم دورات حول العملات المشفرة مما أدى المى زيادة إلمام الجمهور بالعملات المثــفرة ، اصــبحت التكنولوجيا أكثر قيمة من خلال زيادة الطلب على الشـبكة باززدياد عدد مسـتخدميها، كما يوجد الكثير من النشاطات الملحوظة مثل التعدين والتطوير في شراء العملات المشفرة على سبيل المثال ، أكبر بورصة بتكوين في الولايات المتحدة تضيف أكثر من (Coinbase) 100,000 عميل في يوم واحد بعد بورصـة شــيكاغو التجارية حيث أعلنت أنها

ستطلق العقود الآجلة للبيتكوين(Clements,2018:76-77 ) .

Piracy 5.2.1.2.2

"منذ ظهور البيتكوين و انتشـار العملات المشفرة الأخرى، ظهرت مشـكلة كيرة وهي القرصـنة الإلكترونية. وكل اختراق كير في أحد الأظظمة تؤدي إلى تقلب كير

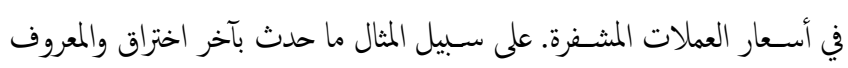
باسم Binance cryptocurrency ، فقد أثر كثيرًا في سعر العملات المشفرة"

. (Maire,2019:15)

Financial Knowledge of 3.1.2.2 المعرفـة المـاليـة لدى المســتيثرين

Investors

يقصـــ بالمعرفة المالية هي فهم المبادئ المالية والمهارات والسـلوكيات اللازمة لأتخاذ قرارات مـالية ســلميمة، وإدارة الاموال بحكمة وفعـالية لوصـــول الى الأمـان العلمي.والمعرفة المالية ضرورية من اجل حاية أنسهم وتحصيها من أيّ انعكات سلبية قد تطرأ نتيجة قصور درايتهم بالشؤون المالية، الثقافة المالية تحوّل الفرد من
تصدر أو تنشئ عملات معدنية جديدة فتبدأ قيمها في الانخفاض، يحدث التضخم عندما يزداد عرض العملة المشفرة، عندما يحدث هذا تصبح العملة المشفرة المعنية أقل ندرة ، ويبدأ الطلب في الانخفاض مما يؤدي إلى الخفاض ســعره وقوته الشرائية، هذه مشكلة الا ان العملات المشفرة لا تتناج إلى القضاء على التضخم (Andrikopoulos et al,2018:5) أنه تخوط جيد ضــد التضـخم، هذا يرجع إلى حقيقة أن العملات المشـفرة لدها إجالي ثابت من 21 مليون عملة معدنية، يعتقد الكثيرون أن هذا يجعل العملات المشـفرة عملة انكماثـية وبالتالي مقاومة للتضخم(Ciaian et al,2018:19) . جادل البعض بأن العملة المشفرة تعاني من معدلات تضخم أعلى بكثير من العملات التياســية، بعض العوامل التي تؤدي إلى التضــــم منها زيادة التكليف الطاقة والإنتاج ، ونمو الدين الوطني اضـافة المى ذلك وجود التضخم كما الظاهرة هو سمة من سمات إدارة التقليدية نظام العملة وهو إلزامي بطبيعته (Jankov,2017:4) . يستشهد Hard Fork تقرير Diar الذي يوضح أن العديد من العملات المشفرة سيكون لها معدلات تضخم عالية جدًا على مدار عام ومع ذلك هذه نظرة قصيرة المدى ، أن العديد من العملات المشــفرة تخطط لتقليل تضـخمها بمرور الوقت .(Yermack,2015:9) للحد من التضـخم تختاج العملات المشـفرة ببسـاطة إلى وضـع حد لعدد الرموز المميزة الجديدة التي تصدرها ومع ذلك ، لا يوجد حل مقياس واحد يناسب الجميع، تعمل كل عملة مشفرة بشكل مختلف، ولكل منها نموذج تضخم مختلف قليلاً. بعض بعري العملات المشـفرة لديها اسـتراتيجيات تضـخم أكثر تفصيلاً من غيرها، تم تصميم Bitcoin للحد من التضـــخم من خلال "نصـــ" المكافأة اي يتم قطع مكافآت الكنالة الخاصة به بشـل دوري إلى النصف مما يجعل التعدين أقل ربجية وبالتالي يثبط بدوره عال المناجم ثم يقل انتاج العملة المشفرة. تستخدم العديد من شركات العملات المشفرة نفس التقنية لمنع التضخم مثل Litecoin - Bitcoin Cash

. (Ciaian et al,2018:35)

Stagnation 3.2.1.2.2 هو حدوث انخفاض عام في الأسعار خلال فترة محددة. على الرغٔ من أن التضخم غالبًا ما يمثل تهديدًا لاقتصاد الدولة، إلا إن الروكد في عالم التمويل في كل يوم يؤدي الى روكد الاقتصـاد العالمي مرهقًا إلى مســتوى قد يتسـبب في ركود جديد. يرى البعض أن قطاع التشــير باكمله سـينهار في حالة الركود ، لأن الناس لن يكتظظوا 


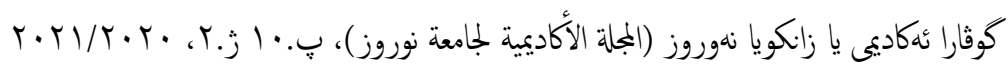

سعر الصرف) .يمكن أن نكون هذه التغييرات مصدر خطر إضافي على المستثمر ، ولكن على نفس المنوال يمكن أن يقلل من الخخاطر بالنسـبة للمس_تثمر ، خخاطر الصرف تساهم بشكل كبير في التقلب الكلي للأمن، ومع ذلك فإن إجلالي المخاطر أقل من مجموع مخاطر السوق والعملات، أظهرت أسعار الصرف وأسواق الأسهم

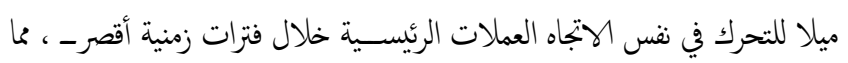

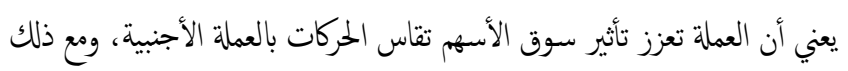
تظهر ننائُ الدراسـات التجريبية الأجنبية أن يتم تعويض مخاطر الصرسف أكثر من فوائد التنويع ، أي يمكن أن تكون انخفاض مخاطر المحفظة. بالإضـافة إلى التنويع ، يككن بالطبع تقليل مخاطر الصرف عن طريق التحوط ، أي إنشاء مراكز قصيرة أو طويلة عن طريق اسـتـخدام العقود الآجلة والعملات الآجلة ، والتي تمثل الأســـــات مراكز طويلة أو قصـيرة مبدئيًا لأدوات الدخل الثابت ، عادةً مع

استحقاق أقل من عام واحد (Bartram \& Dufey,2016:39) (

Gold Price 3.1.2.2

الذهب تثـبه خصـائص العملة المثـفررة من حيث الندرة وقابلية النقل والبنية التحتية ، يككن اعتبار العملة المشــفرة متفوقة على الذهب لأنها( أكثر ندرة من حن الذهب - يمكن نقله مثل ملف رقي - و نظامحا بأكمال يتم إعداده لإنتاج 21 مليون عملة فقط)، وعندما تم الوصــول إلى الحد الأقصى البالغ 21 مليون من العملة المشـفرة. يعتمد توافر الذهب على (دورات العرض والطلب - وارتفاع الطلب على الذهب - حوافز لعحال منابم الذهب)، فيا يتعلق بالبنية التحتية التي تشــير إلى النظام بأكماه الذي يولد ويوزع أحد الأصول - في نظام العملة المشفرة الفعلية ، من أجل إنتاج العملة ، يجب على المرء فقط الاتصـال بـــــنام بيتكوين .يكن لأي شخص متصل بالنظام ويمتلك أبهزة مناسبة المشـاركة في التعدين عن طريق حل مشكلة رياضية صعبة حسابياً (Cocco et al,2919:2) . منس "يعتقد بعض الخبراء أن العملات المشـــفرة يككن ان تحل مكان الحيازات الحقيقية للذهب كملاذ آمن للقيمة عندما تتحول الأمور إلى الأسوأ، ورغً أن مجلس الذهب العالي يرى أن هذه الأصــــل متقلبة للغاية بما لا يؤهلها لأداء تلك المهمة، إلا أن

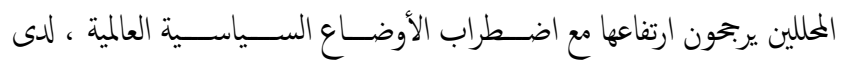

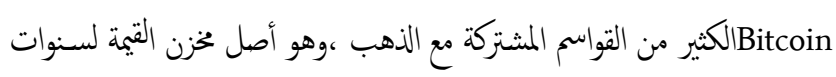
،مقاومة التغيرات التكنولوجية والسـياسـية والاقتصـادية والتغلب على اختبار
مجرد متلقّ إلى متحكم ولاعب فاعل في حياته المالية، أن الفرد المثقت مالياً ولو بالحدّ الأدنى هو أقدر على معرفة ما يريد، وتبيان ما يعرض عليه من منتجات وخدمات واتفاقيات، والمطالبة بمنتجات مالية ومصرفية تتلائم ووضعه الاجتماعي وظروفه الحياتية. أي أن المعرفة المالية لها تأثثر على القرارات التي ســوف تتخذ بشأن الخيارات المتاحة امام المستثمر، كلما زاد الوعي للمسيتمر بالتسوق الألكتروني كلماكان له تأثير ايجابي على قراراته والعكس صحيح كلما قلت المعرفة للمسـتثمر بألأسـتـثار الكلكتروني، للرهن العقاري، البطاقات الألكترونية للأئتان وغيرها من (Olive et al, التقنيات الحديثة المستخدمة له تأثثر سلبي على القرارات المتخذي . 2019:9-10)

\section{External Factors 2.2.2} Macrofinance 1.2.2 المالية الكلية "يلعب متغير سعر الصرف دورًا همًا أيضًا لتحديد سعر العملة المشفرة . ترتبط جميع العملات في جميع أنحاء العالم ببعضــها البعض بجيث يتم تغير عملة واحدة ستؤثر على العملات الأخرى، على الرغ من أن العملة المشفرة ليست مثل العملة القياسـية ، سـوف تتأثر قيمته عندما يتم تغيير أسـعار الفائدة للعملات القياسـية البنوك المركزية أو الحكومة .ترتبط قيمة Cryptocurrency مباشرة بطلبها و جداول العرض ، حيث أن أسعار الفائدة المرتفعة التي تقدها البنوك المركزية سوف تشجع الأشخاص الذين لدهمه عملة قياسية بدلاً من العملات المشفرة بالتالي يكن

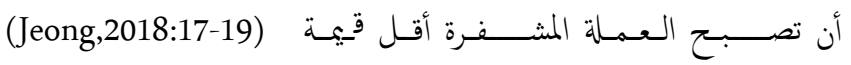
:(Minaudo,2018:5-12)

Interest Rate 1.1.2.2 سعر الفائدة إن قدرة البنك المركزي السيطرة على سعر الفائدة ستتوقف على الطريقة التي يتم بها تكوين العملات المثــفرة، فالأفراد يثــترون العملات المشــفرة مقابل النقود العادية او نظير ودائعهم، وفي كلنا الحالتين ستدخل النقود الى خزينة البنوك وذلك (Dorje "إيداعها في أرصدتهم البنكية وبهذا سيحدث تدفق الى النظام المصرفي . .et al,2019:19)

Exchange Rate 2.1.2.2 سعر الصرف هو عدد الوحدات من عملة قدية أجنبية التي يككن الحصـول عليها مقابل وحدة من العملة الوطنية، اي السـعر النسـبـي لعملة نقدية مقارنة بعملة أخرى، عادة ما تتعرض المحفظة إلى تغييرات غير متوقعة في أســعار صرف العملة المعنية (مخاطر 


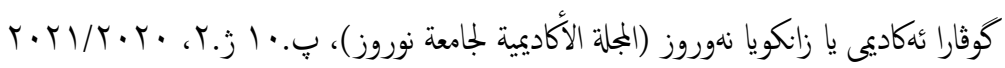

لا يعتمد الطلب على العملات المشفرة وسعرها فقط على العائدات ولكن أيضا على ــ (Jeong,2018:13-15) (Marius,2019: 19) الخخاطر والسيولة

Speculation المضاربات 1.2.2.2

"تعتبر المضاربة من أكثر العوامل التي يكنها أن تؤثر في أسعار العملات المشفرة، فن الممكن أن ترفع المضاربة سعر أحد الأصول إلى مستويات مرتغعة جداً، ومن الممكن أيضاً أن تأخذها الى مستويات منخفضة بشكل حاد، التذبذبات السريعة والقوية من الأمور التي تمِّزٌ العملات المشفرة وتقتح شهيّة المتداولين ، لانها تخلُق فرصًا لكسب الأموال عبر البيع والشراء المدى القصير ، لكنها بطبيعة الحال تاتي بمخاطر أعلى، ويطمح المضــاربون بأن يجنوا ثروات طائلة من خلال التعامل على

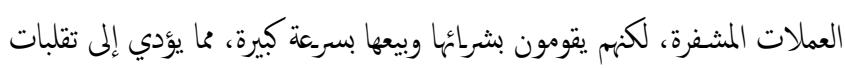

قصير الأجل(Tiwari et al,2018:36) . خلال عام 2017 ارتغ سـعر Bitcoin من حوالي 1,000 دولار إلى ذروة ما يقرب من 20,000 دولار، هذا الارتفاع كان مصـحوبًا بطفرة في تداول سـوق العملات المشفرة ما أدى إلى الازدهام في النظام وتأخير التسوية،"العملة المشفرة هي وسـيلة دفع بدون قيمة جوهرية، تعتمد قيمته على مدى اســتخدامحاكأموال، تكتظ سلسلة الكنل بالمضاربة مما قلل مال العملة المشفرة والثاثثير على سعرها، هذا يثـــر إلى علاقة جديدة بين الاسـتخدام المضـارب والمعاملات للعملات المشـفرة: يمكن زيادة الطلب على المضـاربة عند خفض سـعر الأصـل ، بدلاً من رفعه، في ديسـمبر 2017 التأخير على منصـة Ethereum تفاقتت بسـبب لعبة تداول عبر الإنترنت، أن ازدحام سلسـة الكتل المفلقة تؤدي إلى تفاعلات جديدة بين العملة المشفرة المضاربة والاستخدام النقدي والأسعار من خلال التنافس على مسـاحة محدودة من سـســلة الكتل المفلقة فرض المضـاربين تأثير خارجي على

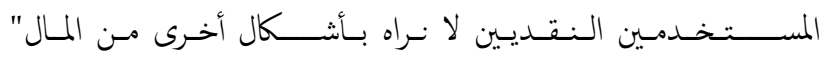

.(Zimmerman,2020:3)

The media 2.2.2.2 وسائل الهلام "أن التغطية الإعلامية الإيجايية أحد العوامل الرئيسـية التي تقود أسـار العملات المشــفرة، تعتبر العوامل الاجتماعية تحمه في تفاعلها وتثأثرها على ســوق العملات المشفرة، وهذه الوسائط تؤثر على الرأي العام ضمن (وسائل الإعلام - الأخبار الصــف - الراديو - التلفزيون - الويب - الهاتف الحمول) كلها وســائل لنقل المعلومات إلى الناشر، حيث تتسـبـب النغطية الإعلامية الإيجابية في إحداث نوع
الزمن أصـبحت فئة أصسول شـائعة جدًا على مر الســنين .بدلاً من ذلك ، فإن Bitcoin جديد ، عمره ثماني سنوات فقط (Baur et al,2018:23).

\section{The Cost of Currencies بلمة العملات 4.1.2.2}

"زيادة الإقبال على العملة المشفرة هو انخفاض قيمة العملات الوطنية في الكثير من الدول على غرار دول منطقة الاتحاد الأوروبي، بجيث أصبحت BTC كملاذ آخر للأفراد والمســتيثرين الذين يبحثون على حلول للحفـاظ على قيــة متلكاتهم أو الاستثمار في أصول جديدة وواعدة.

تؤثر الاضطرابات المالية العلمية على الاقتصـادات والأصـول والعملات في جميع أنخاء العالم.كما تؤثر على المشــاركين في الســـوق وقراراتهم الاســتثخارية، يميل المسيتثرون أكثر خلال الفترات الأزمات إلى إعادة توزيع استثماراتهم على الأصـول التي يتم النظر فيها لتكون ملاذ آمن ، تعتبر العملة المشفرة من أصول الملاذ الآمن إذاكانت دولية يستتمر المستتمرون فيه لتقليل الخسائر خلال فترات الاضطراب المالي .بسـبـب تأثيره على تطوير أســـار صرف العملات Khazal et ) . al,2018:4)

Cryptocurrency markets 2.2.2 أسواق العملات المشفرة "هي مجموعة محركت رئيسية لها تأثير مباثر على سعر العملة المشفرة من العوامل المحددة في ســوق العملات المثـفرة هي ( الشــبية - حجم التجارة - التقلبات المضاربة - معدل العائد وأخرى) من حيث عامل الشعبية ، تستخدم الدراسات السابقة الأنشطة في مجتع العملة المشفرة مثل تردد بحث Twiter ، Google wikapidia على المدى الطويل والقصـير ولها تأثير على جميع العملات المشـفرة حتى لو كانت العوائد المتوقعة على العملة المشفرة أعلى من تلك الموجودة على الأصول الأخرى، تؤدي الزيادة في عائد سوق العملات المشفرة إلى زيادة سعرها في المدى القصير والطويل .التقديرات على المدى القصير للمعاملات أعلى بالمقارنة عع المدى الطويل، يتردد الناس في الاحتفاظ بالعملات المشــفرة عند أختلاف أســعارها يكون التذبذب كير لا يمكن التنبؤ به وهذا تأثيرسـبي، على الرغخ من أن العملة المشـفرة هي اختراع حديث، تعتبر العملة المشـفرة من أصـول المضـاربة وسـلوكها المتعلقة بالأصـول الأخرى أو مؤشرات السـوق، "مســخدم العملة المشـفرة يأخذون في الاعتبار ميزتين إضافيتين بخلاف خصائصها العائد - الخخاطره - السيوله وبالتالي 


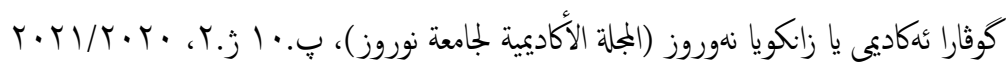

\section{Current Events الأحداث الجارية 1.3.2.2}

"إن الأحداث الجارية هي من أحد الأسباب وأهما والتي تؤثر بصورة غير مباشرة في أسعار العملات المشـفرة وعلى الرغز من أن العملات المشـفرة اقتصـادها غير مرتبط بإحدى الدول، والتي يكن أن تتأثز أســـعارها في حال قامت تلك الدولة بتغيير ما أو دخلت في حالة من الحرب مثلاً، إذا كان هناك تقلبًا بيرًا في أسـعار الدولار مقابل اليوان بسبب الحرب التجارية الجارية،"فبعض الأشخاص ستتفادى التداول على هـا الزوج، ومن الممكن أن يتجهوا إلى الاســتثفار في العملات

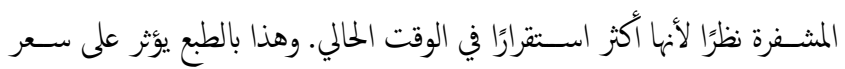
العملات ســواء بالهيوط أو الارتفاع تدخل تصــنيف يطلق عليه fiyat وهذا التصنيف ينطبق على الاستثمارات التي يلجأ الناس لها في حال تذبذب الأسواق العالمية العادية (Vieira,2017:39) (

":Stability 2.3.2.2

أحد أنواع العملات المشفرة ذات الشعبية المتزايدة هي العملات المستقرة. تكون هذه العملات مدعومة بأصـــول يفترض أنها مســـقرة مثل النقد المادي أو الدين الحكوي قصـير الأجل أو غيرها من الأدوات المالية السـائلة عالية الجودة. وهذا

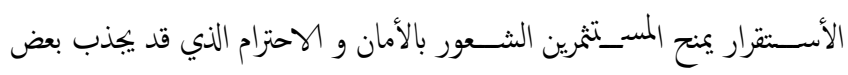
المسيتمرين الذين قد ينظرون إلى الطلب على العملات المشفرة غير المدعومة مثل

(Vieira,2017: 41) (Ethereum - Litecoin - Bitcoin )

Policies and Regulations 3.3.2.2 القوانين واللوائح هي بموعة دافعة رئيسية أخرى لديها تأثير مباثر على سعر سوق العملات المشفرة المحددات في السياسات والمجموعة التنظيمة هي تقنية (التبني) ، التقييد (الحظر) ) ، والنشــاط غير القانوني. منذ بداية عام 2016 ، بدأ سـعر العملات المثـفرة في روسـيا في الظهورحركات تدريجية جنبا إلى جنب مع اليابان والسويد كانت الدول الرئيسية الثلاثة التي تحاول إضفاء الشرعية على استخدام العملة المثــفرة كمملة، عندما اقترحت حكومة كوريا الجنوبية حظرًا على تداول العملات المشفرة أظهر السعر بشكل مكثف .هذا الأثر الضخم للتنظيم الكوري الجنوبي ، وكذلك التهديدات بالحظر في الصـين ، ريماكان السـبب الرئيسي- في عمليات البيع العملات المشـفرة في يناير 2018. اللوائح مثل (تقييد العملة المحلية تعقيد المعاملات - حظر الاســتثمار الأجنبي) بمثابة حظر على تداول العملات المشــفرة"."علاوة على ذلك أشــارت حكومة كوريا الجنوبية من المحتمل أن تخظر
من الضـجيج الإعلامي المعروف أبعاده جيداً، فأن كانت هناك عملة تحصـل على بعض الدعاية السـبية بشـكل عام ان هذه العملة سـوف تتراجع، في هين أنه إذا حصـلت العملة نفسها على دعز كير وتغطية إعلامية جيدة فإن السـعر سـيزداد بالتأكيد، وهذا يعني أن الأسـعار تتأثز بشــة بالعواطف البشرية، كان هذا النمط واضحاً في الأيام الوولى Bitcoin عندما بدأت وسائل الإعلام الرئيسية في تقديم تقرير تضصـيلي عن العملة الجديدة، وفي ظل تزايد التغطية الإعلامية لأخبار وتقلبات اسعار Bitcoin وأخواتها"(Aggarwal et al,2019:392) . Volatility Investors 3:2.2.2 "بعض المس_تثمريين الحاليين في العملات المشـفرة قد عاصروا فترة الألفينات عندما

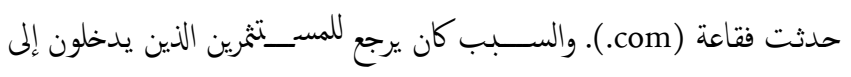
الأســـواق برأس مال ضخم ويحدثون تغييرًا كبيرًا في حركة الأســـوق ولكن لفترة قصيرة"، أن المستمثرون يتداولون في تلك الأسواق بصورة عشوائية وبكميات كيرة ويحدثون تغييرًا ضخمًا في أسعار تلك العملات (Aggarwal et al,2019:392). Cuteness and Audience Acceptance الجاذية وقبول المهور 4.2.2.2 يجد 25 ٪ من المســتثمرين الأوروبيين أن العملات المشـــفرة خالية من الندخل

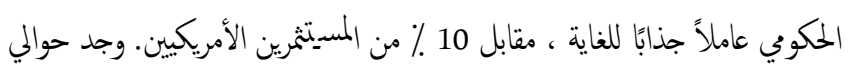
82 \% من المسيتمرين المؤسسيين الأوروبيين شييًّ إيمابيًا بشأن العملات المشفرة ، مقارنة بـــ 74 ٪ من المسيتثرين المؤسسـيين الأمريكيين. إنه انعكس في الرواية المعتادة للأمريكيين المؤيدين للســوق والقطاع الخاص مقابل الأوروبيين الحمبين للحكومة"، ينظر الأوروبيون الآن إلى العملة المشفرة على أنها فئة أصول مرغوبة، لمون

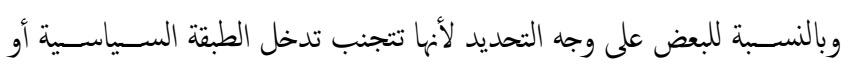

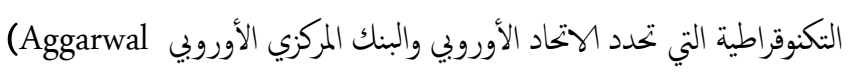
. et al,2019:392)

\section{Political Situations and Events 3.2.2 الأوضاع والأحداث السياسية} "يككن للأحداث والتطورات حول العالم التي لايبدو أنه لا علاقة بالعملات المشــفرة أن تؤثر على الأسـعار ، فعادة ما ينظر إلى هذه الأصـول باعتبارها بديلا

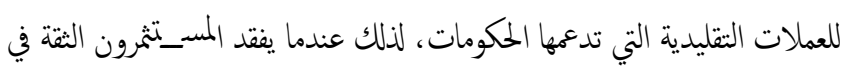

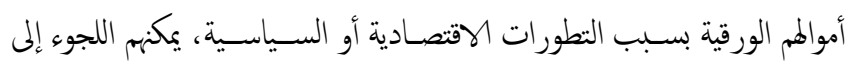
العملات المشفرة ما يؤدي الى رفع الأسعار (Dyhrberg,2016:21) . 


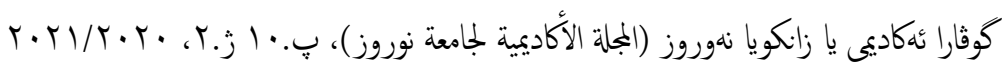

الشركة / المكتب، في هين كانت نسبة (41.3\%) (41.9\%) ( 2 فرد) ( 3 افراد) الذين يعملون في الشركة / المكتب على التوالي . د. وصف عينة البحث حسب ملكية الشركة / المكتب : تبين من التحليل الوصفي لعينة الدراسة بأن كل الشركات / المكاتب تعمل في القطاع الخاص نسبة (100\%) . هـ وصف عينة البحث حسب النشاط الرئيس الشركة / المكتب : تشير نتائج التحليل من بيانات الأستبانة أن نسبة (22.5\%) من الأفراد المبحوثين كان النشاط الرئيس لها التبديل والتحويل العملات ، وجاءت نقس النسبة (38.8) لكل من تبديل العملات و تحويل العملات للأفراد المبحوثين

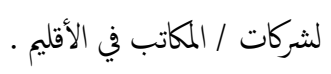
الجدول 1: توزيع عينة البحث حسب البيانات المتعلقة بالشركة / المكتب

\begin{tabular}{|c|c|c|c|c|}
\hline النسبة \% & العدد العد & الفثة & السمة & ت \\
\hline$\% 17.5$ & 28 & قبل 2000 & & \\
\hline$\% 55.6$ & 89 & مابين 2000 - 2010 & تاريخ تأسيس الشركة | & \\
\hline$\% 26.9$ & 43 & مابين 2011 - 2020 & & \\
\hline$\% 40.6$ & 65 & دهوك & & \\
\hline$\% 31.3$ & 50 & اربيل & موقع الثركة / المكتب & \\
\hline$\% 28.1$ & 45 & سليمانية & & \\
\hline$\% 6$ & 1 & 1 & & \\
\hline$\% 41.3$ & 66 & 2 & عدد العاملين في الشركة / & \\
\hline$\% 41.9$ & 67 & 3 & المكتب & \\
\hline$\% 16.3$ & 26 & 4 & & \\
\hline$\% 100$ & 160 & قطاع خاص & & \\
\hline$\% 0$ & 0 & قطاع حكوي & ملكية الشركة / المكتب & \\
\hline$\% 0$ & 0 & قطاع مختلط & & \\
\hline$\% 38.8$ & 62 & تبديل العملات & & \\
\hline$\% 38.8$ & 62 & تحويل العملات & النشاط الرئسى & \\
\hline$\% 22.5$ & 36 & تبديل & & \\
\hline
\end{tabular}

المصدر : من أعداد الباحثة بالأعتاد على أستارة الأستبانة وخرجات البرجية ( SPSS) .

ويكن توزيع الأفراد المبحوثين حسب الخصائص الديموغرافية الأتية وكما مؤشر في الجدول (2) : (2) - (2)

أ. وصف عينة البحث حسب سمة الجنس: يتضح من الجدول المذكور أن نسبة الذكور مثلت ( 100\%) من عينة البحث مقابل نسبة (0\%) للأناث مما
معاملات العملة المشفرة لمهافة استخدامها في غير القانوني أنشطة مثل (غسيل الأموال - القرصنة - السرقات - تمويل الإرهاب - الاتجار غير المشروع بالخحدرات) وما إلى ذلك.بغض النظر عن عدم تجانس شروط كفاءة العملة المشـــفرة ، تتفق

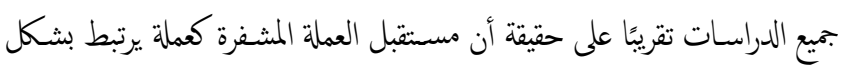

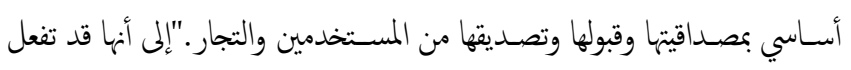
ذلك النظر في بدائل للحظر الكامل ، مثل دفع المسـتتمثرين ضرائب أرباح رأس المال يككن أن تجذب العملة المشـفرة وفرة من الأنشـطة غير القانونية التي لا يككن

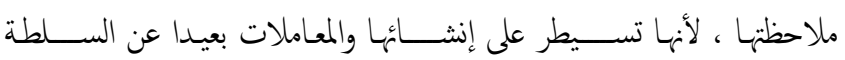
المركزية Cryptocurrency، غير مرتبطة برقابة الحكومة والسـلطة المركزية .(Jeong,2018: 20-22)

\section{3. المور الثالث: الجانب الثطبيقي} 1.3 وصف عينة البحث

تم تخصيص هذا الجانب لوصف خصائص أفراد عينة البحث إذ تم توزيع (160) أستارة على عينة من الشركات / مكاتب Forex وتبادل العملات في الأقليم للعملات المثفرة ، حيث كانت الحصيلة النهائية مساوية إلى (160) أستارة صالحة للتحليل وبذلك بلغت نسبة الأستجابة ( 100\%) . تم توزيع الأفراد المبحوثين حسب الخصائص الأتية وكما مؤشر في الجدول (1): أ. وصف عينة البحث حسب تاريخ تأسيس الشركة / المكتب : يتضح من الجدول (1) أن اعلى نسبة ( 55.6\%) هي للفئة مابين (2000-2010) كتارين تأسيس الشركة / المكتب من عينة البحث و نسبة (26.9\%) للفئة مابين (2011-2020) وكانت الفئة (قبل 2000) مثلت اقل نسبة Forex (17.5) وتبادل العملات الأغلبية تأسست مابين (2000-2010) في الأقليم. ب. وصف عينة البحث حسب موقع الشركة / المكتب : بلغت أعلى نسبة من الشركات / المكاتب عينة الدراسة لمحافظة دهوك بنسبة (40.6\%)، ونسبة (31.3\%) للشركات / المكاتب عينة الدراسة لمحافظة في محفظة اربيل ، بينا مثلت نسبة (28.1\%) للأفراد في محافظة السليانية . ج. وصف عينة البحث حسب عدد العاملين في الشركة / المكتب : تشير نتائج التحليل من بيانات الأستبانة أن اقل نسبة مثلت (6\%) لفرد واحد فقط يعمل في الشركة / المكتب و نسبة (16.3\%) لـ (4 افراد) يعملون في 


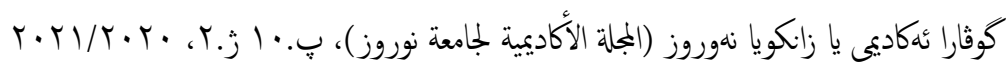

أفراد العينة هم من حملة الشهادات الأكاديمية مما يسهم في إمكانة فهم المستجيب العبارات وإدراكها وأستيعاب مضامين أستارة الأستبيان والإجابة عنها بمستويات عالية من الموضوعية .

ز. وصف عينة البحث حسب سمة التخصص : يتضح من الجدول (2) أن أعلى نسبة من الأفراد المبحوثين هم من حملة شهادة بكلوريوس بنسبة (65.5\%) حيث كانت اعلى نسبة لاختصاص كلية الادارة والاقتصاد بنسبة (28.8\%) ومن ثخ تلنها كل من كلية تربية الاساس وكلية العلوم الأنسانية بنسبة (9.4\%) (8.1\%) على التوالي، وجاءت نفس النسبة (3.1\%) لكل من كلية التربية الرياضية وكلية العلوم ، ونسبة (5.6\%) كانت من نصيب كلية القانون وكلية الزراعة، في حين جاءت كلية الهندسة بالمرتبة الاخيرة بنسبة (1.9\%) من الأفراد المبحوثين من حملة شهادة البكلاريوس، وكانت نسبة (18.8\%) للأفراد المبحوثين ليس لديهم تخصص، في حين نسبة (15.6\%) مثلت اختصاص المعاهد، اعلى نسبة كانت للمعهد إدارة الاععال بنسبة (4.4\%) واقل نسبة كانت (1.9\%) لمعهد محاسبة من الافراد المبحوثين ، وجاءت نفس النسبة (3.1\%) لكل من معهد الصناعة ومعهد النفط ومعهد ميكنيك من الافراد المبحوثين . وصف عينة البحث حسب عدد الدورات الثدريبية المثعلقة بالعملات المشفرة : تشير نتائُ التحليل من بيانات الأستبانة أن نسبة (100\%) من الأفراد المبحوثين لم يتلقوا اي دورة متعلقة بالعملات المشفرة للمكاتب والشركات في · الاقليم

2.3 اختبارات أداة القياس

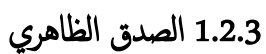

تضمتت هذه المرحلة إعداد استخارة الأستبانة بصينها الأولية pilot study كسح أولي لــــ (30 شركات/مكاتب) تبادل العملات في مدينة دهوك فقط من أجل معرفة مدى ملائمة الأستبانة وفقراتها لموضوع الدراسـة.ومن ثُ غُضِتـت على عدد من الخبراء في مجالات العلوم الأدارية و المالية المصر_فية لإبداء آرائهم من حيث صياغة العبارات ودقتها في قياس متغيرات الدراسـة وأختبار فرضياتها، وتم الأخذ بأرائه باختصار عـدد مـن العبـارات أو حسذفها او دمج عدد منها وإعادة صياغة عدد أخر من العبارات وبما يحقق صــدق الظاهري للعبارات قيد الدراسـة، ثم تم
يعطي مؤشر أولي على أن كل الشركات / مكاتب Forex وتبادل العملات

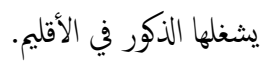
ب. وصف عينة البحث حسب سمة العمر : بلغت أعلى نسبة من الأفراد المبحوثين ضمن الفئة العمرية (مابين 31 - 40 سنة) وبنسبة (40.4\%)، في حين أن الفئة العمرية (41 سنة فاكثز) وبنسبة بلغت (35.6\%) ، بينا مثلت نسبة (24.4\%) الأفراد الذين كانت فئاتهم العمرية (اقل من 35 سنة)

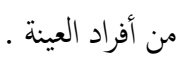

ج. وصف عينة البحث حسب مدة المدمة في الوظيفة :ينيبن من الجدول (2) أن نسبة الأفراد (30.6\%) الذين كانت مدة خدمتهم في الوظيفة الوظيفة تتزاوح بين (10 سنوات فأكثر) اما الأفراد الذين كانت مدة خدمتهم مابين (7 - 9 ) سنوات كانت نسبته (26.3\%) في حين كانت نسبة (23.1\%) للأفراد الذين كانت مدة خدمتهم في الوظيفة مابين (4 - 6 ) سنوات ، وجاءت نسبة (20.0\%) بالمرتبة الأخيرة للأفراد المبحوثين الذين كانت مدة خدمتهم مابين (1 - 3 ) سنوات . د. وصف عينة البحث حسب سمة المنصب الوظيفي : بلغت نسبة الأفراد المبحوثين الذين يشغلون منصب مدير (59.4\%) من المبحوثين وبنسبة (40.6\%) ضمن المنصب الوظيفي (محاسب) او تحت تسمية مدير مالي. . هـ وصف عينة البحث حسب مدة الخدمة في المنصب الوظيفي : تشير نتأُج التحليل من بيانات الأستبانة أن نسبة (28.1\%) ونسبة (26.3\%) من الأفراد المبحوثين كانت مدة خدمتهم في المنصب الوظيفي تتراوح بين (7-9 سنوات) و (10 سنوات فأكثر) على التوالي، أما الأفراد الذين كانت نسبته (23.1\%) هم الافراد الذين كانت مدة خدمتهم في المنصب الوظيفي ما بين (1-3 سنوات) ،وقد جاءت بالمرتبة الأخيرة الأفراد الذين كانت مدة خدمتهم في المنصب الوظيفي (1 - 3 ) سنوات وبنسبة (22.5\%) . و. وصف عينة البحث حسب سمة الشهادة : يتضح من الجدول (2) أن أعلى نسبة من الأفراد المبحوثين هم من حملة شهادة بكالوريوس بنسبة (63.7\%) ، في حين مثلت نسبة (18.8\%) هم من حملة شهادة إعدادية فأقل من الأفراد المبحوثين وكانت النسبة (15.0\%) هم من حملة شهادة دبلوم معهد ، اما المرتبة الاخيرة جاءت فئة الافراد من حملة شهادة دراسات عليا وبلغت النسبة (2.5\%)، وهذه المعطيات تعطي لتا تصوراً بأن الغالبية العضمى من 


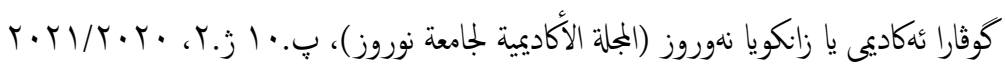

أ. قياس الشمولية: تضمنت هذه المرحلة عرض استمارة الأستبانة وطرحما على الســادة الخبراء لمعرفة مدى شمولية العبارات حيث تم أضــافة عدد من

العبارات وحذف البعض الآخر ، فأصبحت الاستبيان بصينتها الهائية . ب. قياس الثبات: أسـتخراج قيمة ثبات أسـتمارة الأسـتبانة عن طريق معامل الثبات (Cronbach-Alpha)، تم أســتخراج معامل الثبات لعبارات الأســتبانة لعينة الأولية (30 شركة/مكتب) بلغت (0.991)، ومعامل الثبات لعينة الثــاملة (160شركة/مكنب ) حيث بلغت (0.993) وفق معاملة الفا كرونباخ وهي درجة عالية لتقيم ثبات الأستبانة . القوة التمينة : ولأجل اسـتشخراج القوة التميزية للفقرات تم تطبيق المقياس على عينـة بلغـت (160شركات/مكاتـب) تبـادل العملات، وبعـد جمع الاستمارات وتصحيحها بإعطاء درجة كلية لكل استمارة رتبت الاستمارات حسب الدرجات تنازلياً من أعلى درجة إلى أدنى درجة، ثم أخذت نسـبة (27\%) من الاسـتمارات التي حصـلت على أعلى الدرجات لتمثل المجموعة العليا وبلغ عددها ( 43 ) استتارة و(27\%) من الاستخارات التي حصلت على أقل الدرجات لتمثل الجمموعة الدنيا وبلغ عددها ( 43 ) اسـتمارة ، ذلك

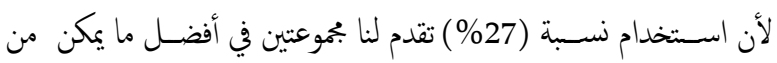

الحجم والتيزيز بحسب أدبيات التياس والتقويم (الكناني، 2014: 57 ) . ولحسـاب القوة التمييزية لكل فقرة من فقرات المقياس، اسـتخدمنا الاختبار (T)

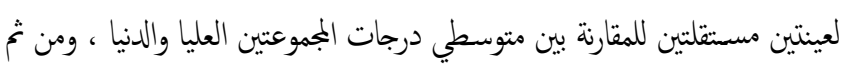
مقارنة القيمة (T) المحســوبة لكل فقرة من فقرات المقياس مع القيمة (T) الجدولية البالغة (2,35) عند مستوى دلالة (0,05) ودرجة حرية تسـأوي (84) بمتوسط حسـابي (53.98) وأخراف معياري (0.152) و (Sig 0.000 ). أن القيم (T) المحســوبة أكبر من القيمة (T) الجدولية، أي أن القيم (T) المحســـوبة لها تأثير معنوي،والجدول (3) يبين ذلك . الجدول (3): أختبار التائي لثبات المقياس

\begin{tabular}{|c|c|c|c|c|c|c|}
\hline Sig & $\mathrm{DF}$ & Mean & St.D & قالجملية T & قلمسمة T & المتغير \\
\hline 0.000 & 84 & 53.98 & 0.152 & 2.35 & -8.152 & المبالعملات المشفة المالية \\
\hline
\end{tabular}

التوزيع على عينة اشمل وتضــمنت شركات / مكاتب والبالغة عددها (160 الشركات/مكاتب) في محفظات الثلاث (دهوك، اربيل، سليمانة). الجدول (2): توزيع عينة البحث حسب خصائص الديوغرافية المتعلقة بالشركة / المكتب

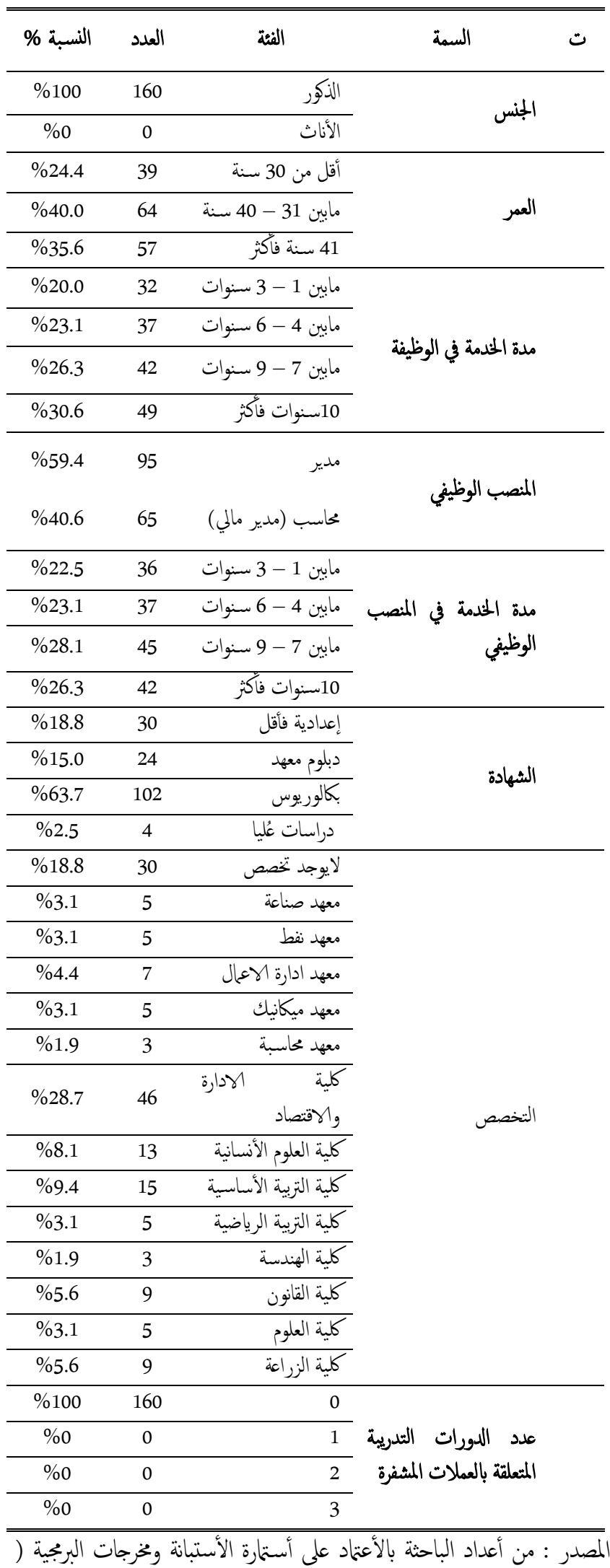

. (SPSS 


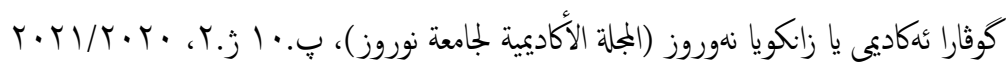

أفراد العينة ليس لديهم معرفة تجاه مجال المعرفة المالية بالعملات المشفرة والرقية بآلية عملها وأنواعها، وتأثير الأوضاع السياسية والسياسات النقدية والمخاطر المصاحبة على سعر السوق للعملات المشفرة .

أما على مستوى الجزئي لكل مؤشر من مؤشرات قياس فقد بلغ مؤشر ( X7

X8 - أعلى نسبة أتفاق على مستوى العبارات والتي بلغت (14.4\%)، وبوسط حسابي بلغ (1.48) (1.54) وانحراف معياري (1.104) (1.258) على التوالي، ما يدل على تأثير عملية تعدين العملات المشفرة سلباً وبشكل كير على تداولها، وتثاثير التغيرات الاقتصادية مثل التضخم والركود الأقتصادي والعرض والطلب بشكل مباشر على أسعار العملات المشفرة، أما أقل قيمة فقد كانت للمؤشر (X17) والتي بلغت (6.9)، أذ بلغ الوسط الحسابي لها (1.37) وبانحرف معياري (0.962) والتي تبين عدم امتلاك وعي ومعرفة كافية بأنظمة الدفع والتحويل بالعملات المشفرة

وخدمات الدئ وتسوية النزاعات وغيرها.

الجدول 5: وصف أراء عينة البحث تجاه عبارات المعرفة المالية

\begin{tabular}{|c|c|c|c|c|c|c|c|c|c|c|c|c|}
\hline \multirow{2}{*}{ اللماري } & \multirow{2}{*}{ المسالي } & \multicolumn{2}{|c|}{ لالامت ششة } & \multicolumn{2}{|c|}{ لآثن } & \multicolumn{2}{|c|}{$4 k$} & \multicolumn{2}{|c|}{ أهن } & \multicolumn{2}{|c|}{ أهن هشة: } & \multirow{2}{*}{ البراز } \\
\hline & & النبة & التكرار & السبة & التكرار & النبة & الثكرار & النبة & التكرار & النبة & التكرار & \\
\hline 1.081 & 1.48 & 83.1 & 133 & 0.6 & 1 & 2.5 & 4 & 13.1 & 21 & 0.6 & 1 & $\mathbf{X} 1$ \\
\hline 1.057 & 1.45 & 83.1 & 133 & 2.5 & 4 & 1.9 & 3 & 11.3 & 18 & 1.3 & 2 & $\mathrm{X}_{2}$ \\
\hline 0.987 & 1.42 & 83.1 & 133 & 3.1 & 5 & 2.5 & 4 & 11.3 & 18 & $\cdot$ & $\cdot$ & $\mathrm{X}_{3}$ \\
\hline 1.091 & 1.44 & 83.8 & 134 & 3.1 & 5 & 3.8 & 6 & 4.4 & 7 & 5.0 & 8 & $\mathrm{X}_{4}$ \\
\hline 1.165 & 1.48 & 83.8 & 134 & 1.9 & 3 & 2.5 & 4 & 6.3 & 10 & 5.6 & 9 & $\mathrm{X}_{5}$ \\
\hline 1.195 & 1.51 & 83.1 & 133 & 0.6 & 1 & 1.9 & 3 & 8.8 & 14 & 5.0 & 8 & X6 \\
\hline 1.104 & 1.48 & 83.1 & 133 & 1.3 & 2 & 1.3 & 2 & 13.1 & 21 & 1.3 & 2 & $\mathrm{X}_{7}$ \\
\hline 1.258 & 1.54 & 83.1 & 133 & 1.9 & 3 & 0.6 & 1 & 6.9 & 11 & 7.5 & 12 & $\mathrm{X8}$ \\
\hline 1.138 & $\begin{array}{ll}1.47 \\
\end{array}$ & 83.8 & 134 & 2.5 & 4 & 1.3 & 2 & 8.1 & 13 & 4.4 & 7 & $\mathrm{Xg}$ \\
\hline 1.109 & 1.46 & 83.8 & 134 & 2.5 & 4 & 1.9 & 3 & 8.1 & 13 & 3.8 & 6 & $\mathrm{X} 10$ \\
\hline 1.163 & 1.49 & 82.5 & 132 & 1.9 & 3 & 3.8 & 6 & 5.6 & 9 & 5.6 & 9 & $\mathrm{X} 11$ \\
\hline 1.018 & 1.41 & 84.4 & 135 & 1.9 & 3 & 3.8 & 6 & 8.1 & 13 & 1.9 & 3 & $\mathrm{X}_{12}$ \\
\hline 1.081 & 1.46 & 83.1 & 133 & 2.5 & 4 & 2.5 & 4 & 9.4 & 15 & 2.5 & 4 & $\mathrm{X}_{13}$ \\
\hline 1.051 & 1.44 & 83.1 & 133 & 2.5 & 4 & 3.1 & 5 & 9.4 & 15 & 1.9 & 3 & $\mathrm{X} 14$ \\
\hline 1.129 & $\begin{array}{l}.47 \\
\end{array}$ & 83.8 & 134 & 0.6 & 1 & 3.8 & 6 & 7.5 & 12 & 4.4 & 7 & X15 \\
\hline 1.030 & \begin{tabular}{ll|}
1.41 \\
\end{tabular} & 84.4 & 135 & 3.1 & 5 & 3.1 & 5 & 6.3 & 10 & 3.1 & 5 & $\mathrm{X} 16$ \\
\hline 0.962 & 1.37 & 84.4 & 135 & 4.4 & 7 & 4.4 & 7 & 3.8 & 6 & 3.1 & 5 & $\mathrm{X} 17$ \\
\hline 0.974 & \begin{tabular}{ll|}
1.41 \\
\end{tabular} & 83.1 & 133 & 2.5 & 4 & 5.0 & 8 & 8.8 & 14 & 0.6 & 1 & $\mathrm{X} 18$ \\
\hline 1.121 & 1.47 & 83.8 & 134 & 1.3 & 2 & 3.1 & 5 & 8.1 & 13 & 3.8 & 6 & X19 \\
\hline 1.182 & 1.50 & 83.8 & 134 & 0.6 & 1 & 2.5 & 4 & 8.1 & 13 & 5.0 & 8 & $X_{220}$ \\
\hline 1.143 & 1.46 & 84.4 & 135 & 1.9 & 3 & 1.9 & 3 & 6.9 & \begin{tabular}{ll|}
11 \\
\end{tabular} & 5.0 & 8 & X21 \\
\hline 1.127 & 1.46 & 83.1 & 133 & 2.5 & 4 & 1.9 & 3 & 7.5 & 12 & 4.4 & 7 & $\mathrm{X} 22$ \\
\hline \multirow[t]{2}{*}{1.154} & 1.47 & 83.1 & 133 & $\begin{array}{l}4.4 \\
\end{array}$ & 7 & 1.3 & 2 & $\begin{array}{l}5.0 \\
\end{array}$ & 8 & 6.3 & 10 & $\mathrm{X}_{23}$ \\
\hline & & 83.51 & & 2.17 & & 2.63 & & 8.08 & & 3.73 & & المعل \\
\hline 1.100 & 145 & \multicolumn{4}{|c|}{85.67} & \multicolumn{2}{|c|}{2.63} & \multicolumn{4}{|c|}{11.81} & الكلي \\
\hline
\end{tabular}

المصدر : من أعداد الباحثة وفق نتائُ التحليل الوحصائي بالاعتاد على مخرجات البرجية (SPSS).

\subsection{3 وصف آراء العينة تجاه عبارات التوجمات المستقبلة العالمية للعملات المثفرة}

تشير معطيات التهليل الواردة في الجدول (6) إلى إجابات المبحوثين والتوزيعات التكرارية والأوساط الحسابية والأنخرافات المعيارية للإجابات تجاه العبارات (-X24 X43 الخاصة بوصف عينة الدراسة تجاه التوججات المستقبلية العلمية للعملات المشفرة التي تميل بإتجاه عدم الأتفاق بمستويات عالية، أذ تشير النسب بحسب المؤشر الكلي الى (84.2\%) من افراد عينة الدراسة عدم اتفاقهم على مضمون هذه
3.3 مقياس البحث

تم استخدام مقياس (ليكرت) الحماسي المتكون من (5) بدائل للقرار لما يوفره من مزايا في عملية القياس ، حيث استخدمت (54) عبارة تتضمن (23) عبارة بالمعرفة المالية بالعملات المشفرة والرقية و(20) عبارة للتوجهات المستقبلية العالمية للعملات المشفرة و (11) عبارة لطريقة التداول بالعملات المشفرة وكما موضح في الجدول الأتي: الجدول 4 : توزيع عبارات البحث وفق مقايسها

\begin{tabular}{|c|c|c|c|c|}
\hline مجموع الفقرات & & رمّ العبارة & أسم المثغير & ت \\
\hline \multirow{3}{*}{54} & من 1 لغاية 23 & المعرفة المالية & & \\
\hline & من 24 لغاية 43 & المستجنجلية & المشفلات & 1 \\
\hline & من 44 لغاية 54 & طريةة النداول & & \\
\hline
\end{tabular}
المصدر : من إعداد الباحثة

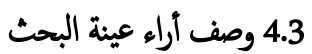
يتضمن المبحث الحالي وصف لأراء المبحوثين وذلك بتحليل البيانات المتعلقة بها، والتي تتم فيه أستخدام التوزيعات التكرارية والنسب المئوية والأوساط الحسابية والأخرافات المعيارية، من خلال آراء عينة الدراسة تجاه المتغير العملات المشفرة ( المعرفة المالية بالعملات المشفرة والرقية - التوجهات المستقبلة العالمية للعملات المشفرة - طريقة التداول العملات المشفرة للشركات / المكاتب) قد تراوحت بين الأتفاق التام وعدم الأتفاق ومحايد وذلك كما يلي :

\subsection{3 وصف آراء العينة تجاه عبارات المعرفة المالية بالعملات المشفرة والرقية :}

تشير معطيات التحليل الواردة في الجدول (5) إلى إجابات المبحوثين وعلى المستوى الكلي للشركات / المكاتب المبحوثة والتوزيعات التكرارية والأوساط الحسابية والأخرافات المعيارية للإجابات تجاه العبارات (X1-X23) الخاصة بوصف عينة الدراسة تجاه المعرفة المالية بالعملات المشفرة والرقية، التي تميل بإتجاه عدم الأتفاق بمستويات عالية، أذ تشير النسب بحسب المؤشر الكلي الى (85.67\%) من افراد عينة الدراسة عدم اتفاقمم على مضمون هذه العبارات في حين بلغت نسبة محايد (2.63\%)، أما نسبة الأتفاق فقد بلغت (11.81\%)، وجاء بوسط حسابي مقداره (1.45) وانحراف معياري مقداره (1.100). مما يعطي دلالة أولية على أن 


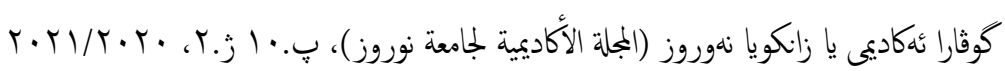

الى (100\%) من افراد عينة الدراسة عدم اتفاقهم على مضمون هذه العبارات في حين بلغت نسبة محايد (0\%)، أما نسبة الأتفاق فقد بلغت (0\%)، وجاء بوسط حسابي مقداره (1.00) وانخراف معياري مقداره (0.000). مما يعطي دلالة أولية على أن أفراد العينة لعدم وجود تداول بالعملات المشفرة للشركة / المكتب في الأقليم سواءًً كانت الطريقة المباشرة او طريقة غير مباشرة .

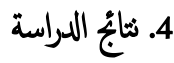

لتحديد مسـتوى المعرفة المالية والرؤية المسـتقبلية للعملات المشـفرة لدى أفراد الدراسة، تم حساب متوسط الدرجات ومن ثم مقارنتها مع المتوسط الفرضي لكل متغير بعد أســتخدام الاختبار (T) لعينة واحدة فتبين من النتائُ أن هناكُ دال أحصـائيا بين المتوســط المحسـوب والمتوسـط الفرضي للمعرفة المالية والتوبجات المســتقبلة للعملات المشــفرة، بينها لم يظهر فرق دال لتداول العملات المشــفرة

$$
\text { للشركات/مكاتب في الأقليم، كما موضح في الجدول (7) . }
$$
الجدول 7: دلالة الفرق بين الوسط الحسابي والوسط الفرضي للمعرفة المالية للعملات المشفرة (no.160)

\begin{tabular}{|c|c|c|c|c|c|c|}
\hline $\begin{array}{c}\text { Sig } \\
(0.05)\end{array}$ & T.test & DF & St.D & الفرضي & الحسابي & المثغير \\
\hline 0.000 & 17.625 & 159 & 1.047 & 108 & 1.45 & المالمرفة \\
\hline 0.000 & 16.853 & 159 & 1.126 & 108 & 1.50 & المستثلية \\
\hline- & - & - & 0.000 & 108 & 1 & العملات \\
\hline
\end{tabular}

المصدر : من أعداد الباحثة وفق نتاجُ التحليل الاحصائي بالاعتماد على خرجات البرجية ( SPSS). يوجد فرق دال عند مستوى دلالة (0.05) بين الوسط المسسوب للمعرفة المالية بالعملات المشـفرة (1.45) درجة والوســ الفرضي البالغ (108) درجة بانحراف معياري (1.047) ودرجة حرية (159) ، مما يدل على عدم وجود المام وادراك المعرفة المالية بالعملات المشفرة لدى اصحاب ومدراء الشركات ومكاتب التداول في

يوجد فرق دال عند مســتوى دلالة (0.05) بين الوســ المسسـوب التوجحات المستقبلية للعملات المشفرة (1.50) درجة والوسط الفرضي البالغ (108) درجة بانراف معياري (1.126) ودرجة حرية (159) ، مما يدل على عدم وتوجحات ورؤية مسـتقبلية بالعملات المشــفرة لدى اصحاب ومدراء الثرسكات ومكاتب

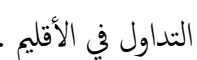

العبارات في حين بلغت نسبة محايد (2.67\%)، أما سبة الأثفاق فقد بلغت (13.39\%)، وجاء بوسط حسابي مقداره (1.51) وانحراف معياري مقداره (1.28). ما يعطي دلالة أولية على أن أفراد العينة ليس لديهم رؤية مستقبلية بإتجاه العملات المشفرة وزيادة عددها وانواعها والأستثمار بها وجمال تطوير التقنية الرقية وذلك بوجود تقانة المعلومات ومتاجر الألكترونية. أما على مستوى الجزئي لكل مؤشر من مؤشرات قياس فقد بلغ مؤشر (X31) أعلى نسبة أتفاق على مستوى العبارات والتي بلغت (15.6\%)، وبوسط حسابي بلغ (1.51) وانخراف معياري (1.176) ، مما يدل على وجود العملات المشفرة يشكل حافزاً على تحويل الأموال دون الحاجة إلى امتلاك حساب مصرفي، أما أقل قيمة فقد كانت للمؤشر (X32) والتي بلغت (9.4\%)، أذب بلغ الوسط الحسابي لها (1.56) وبانخرف معياري (1.263) والتي تبين وجود تطبيقات وبرامج الكترونية للمنصات الرئيسية مثل Apple Ios ،Android لتطوير طرق آمنة وسهلة لالإرسال واستلام الأموال.

الجدول 6: وصف أراء عينة البحث تجاه عبارات التوجحات المستقبلية العالمية للعملات المشفرة

\begin{tabular}{|c|c|c|c|c|c|c|c|c|c|c|c|c|}
\hline \multirow{2}{*}{ المارئريا } & \multirow{2}{*}{ المسائ } & \multicolumn{2}{|c|}{ لااثق شيلة } & \multicolumn{2}{|c|}{ لעام } & \multicolumn{2}{|c|}{$+k$} & \multicolumn{2}{|c|}{ أهن } & \multicolumn{2}{|c|}{ أثن يشلة } & \multirow{2}{*}{ البارز } \\
\hline & & النسة & الككارا & النبة & التكرار & النبة & التكرار & النسبة & التكرار & النسة & التكرار & \\
\hline 3.547 & 1.74 & 83.1 & 133 & 1.3 & 2 & 2.5 & 4 & 9.4 & 15 & 3.8 & 6 & $\mathrm{X}_{24}$ \\
\hline 1.110 & 1.46 & 84.4 & 135 & 0.6 & 1 & 1.9 & 3 & 10.6 & 17 & 25 & 4 & $\mathrm{X}_{25}$ \\
\hline 1.099 & 1.48 & 83.8 & 134 & - & - & 2.5 & 4 & 12.5 & 20 & 13 & 2 & $\mathrm{X} 26$ \\
\hline 1.149 & 1.48 & 83.8 & 134 & 1.9 & 3 & 25 & 4 & 6.9 & 11 & 5.0 & 8 & $\mathrm{X} 27$ \\
\hline 1.233 & 1.53 & 83.1 & 133 & 1.3 & 2 & 1.3 & 2 & 8.1 & 13 & 6.3 & 10 & $\mathrm{X} 28$ \\
\hline 1.236 & 1.53 & 83.1 & 133 & 1.3 & 2 & 1.3 & 2 & 8.1 & 13 & 6.3 & 10 & $\mathrm{X}_{22}$ \\
\hline 1.171 & 1.51 & 83.1 & 133 & 0.6 & 1 & 3.1 & 5 & 8.8 & 14 & 4.4 & 7 & $\mathrm{X}_{30}$ \\
\hline 1.176 & 1.51 & 83.1 & 133 & 1.3 & 2 & - & - & 12.5 & 20 & 3.1 & 5 & $\mathrm{X}_{31}$ \\
\hline 1.263 & 1.56 & 83.1 & 133 & - & - & 1.3 & 2 & 3.1 & 5 & 6.3 & 10 & $\mathrm{X} 32$ \\
\hline 1.176 & 1.51 & 83.1 & 133 & 0.6 & 1 & 1.9 & 3 & 10.6 & 17 & 3.8 & 6 & $\mathrm{X}_{33}$ \\
\hline 1.166 & 1.49 & 83.1 & 133 & 1.9 & 3 & 25 & 4 & 75 & 12 & 5.0 & 8 & $\mathrm{X} 34$ \\
\hline 1.138 & 1.49 & 83.1 & 133 & 0.6 & 1 & 3.1 & 5 & 10.0 & 16 & 3.1 & 5 & X35 \\
\hline 1.075 & 1.47 & 83.1 & 133 & - & - & 5.6 & 9 & 9.4 & 15 & 1.9 & 3 & X36 \\
\hline 1.160 & 1.50 & 83.1 & 133 & 0.6 & 1 & 3.8 & 6 & 8.1 & 13 & 4.4 & 7 & X37 \\
\hline 1.069 & 1.46 & 83.1 & 133 & 0.6 & 1 & 5.0 & 8 & 9.4 & 15 & 1.9 & 3 & $\mathrm{X} 38$ \\
\hline 1.166 & 1.49 & 83.1 & 133 & 1.3 & 2 & 4.4 & 7 & 5.6 & 9 & 5.6 & 9 & X39 \\
\hline 1.187 & 1.52 & 83.1 & 133 & - & - & 3.1 & 5 & 9.4 & 15 & 4.4 & 7 & $\mathrm{X}_{40}$ \\
\hline 1.187 & 1.52 & 83.1 & 133 & 0.6 & 1 & 1.3 & 2 & 11.3 & 18 & 3.8 & 6 & $\mathrm{X}_{41}$ \\
\hline 1.182 & 1.51 & 83.1 & 133 & 1.3 & 2 & 2.5 & 4 & 8.1 & 13 & 5.0 & 8 & $\mathrm{X} 42$ \\
\hline \multirow[t]{2}{*}{1.155} & 1.50 & 83.1 & 133 & 1.3 & 2 & 1.3 & 2 & 11.3 & 18 & 3.1 & 5 & $\mathrm{X}_{A 3}$ \\
\hline & & 83.2 & & 1.06 & & 267 & & 9.03 & & 4.36 & & المعل \\
\hline 1.28 & 1.51 & \multicolumn{4}{|c|}{842} & \multicolumn{2}{|c|}{2.67} & \multicolumn{4}{|c|}{13.39} & الكلى \\
\hline
\end{tabular}

المصدر : من أعداد الباحثة وفق نتائُ التحليل الوحصائي بالاعتاد على مخرجات البرجية ( SPSS).

\subsection{3 وصف آراء العينة تجاه عبارات طريةة الثداول بالعملات المشفرة للثركة /}

.2 المكتب للأقليم

تشير معطيات التحليل إلى إجابات المبحوثين والتوزيعات النكرارية والأوساط الحسابية والأخرافات المعيارية للإججابات تجاه العبارات (X54-X44) الخاصة بوصف عينة الدراسة تجاه طريقة التداول بالعملات المشفرة للشركة / المكتب التي تميل بإتجاه عدم الأثفاق بمستويات عالية، أذ تشير النسب بحسب المؤشر الكلي 


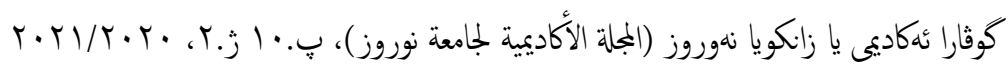

تشجيع الجهات المعنية في الأقليم نو تطبيق تداول العملات المشفرة لتقليل الثاثيرات السـلبية التي تواجه السـياســة التقدية ويكون بديلا عن بعض

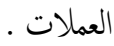

وجود تنظيم قانون دولي لمسـألة أصـدار العملة المشـفرة والعمل على مكافة الجرائم التي تحدث من خلال تداولها والتي تسـاهم في خلق المشــاكل مثل

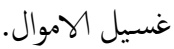
من الضرورة أن تسـام المؤسسـات النعلمية (الجامعات والمعاهد) وخاصة الأكادميين والباحثين في تخصصات الإدارة المالية، العلوم المالية والمصرفية، المحاسبة والأقتصاد بضرورة الأسهام في نشر-توعية التبادل بالعملات بشكل عام والعملات المشفرة بشكل خاص وزيادة المعرفة المالية للمتعاملين بها من خلال برامج التدريب وورش العمل مشتركة .

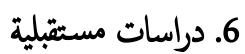
يفرض علينا أستنتاجات هذه الدراسة بضرورة الأستمرار في دراسة العملات المشفرة بشكل أثمل، حيث يعد الموضوع الأكثر أثارة على مستوى العالم، حيث وصـل ســر Bitcoin الواحد الى (53.700 أمريكي) بتاريخ 2021/3/16 ـ ووصـل عدد العملات المثــفرة بكل صـيغها ومعادلاتها وطريقة تصـيمها الى قرابة (8800) عملة مشفرة في العراق بشكل عام وفي

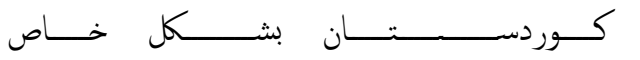

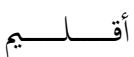
https://coinmarketcap.com/currencies/bitcoin/historic) al-data/ تقترح الدراسة الحالية ضرورة الأسيتمرار في الدراسة مستقبلاً في العناوين والمواضيع المتعلقة بالعملات المشفرة كما يلي : O قياس مســتوى الأسـتـثار بالعملات المثــفرة في الأقليم من قبل · المستثمرين O دراسة مدى تقبل وجود عملة مشفرة كوردية في الأقليم . O أثر العملات المشفرة في تقليل مخاطر المحافظ الأستثارية.

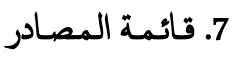
1.7
عدم وجود فرق دال عند مسـتوى (0.05) بين الوسطط الحسـابي (1) للتداول .3 العملات للشركات والمكاتب في الأقليم والوسط الفرضي البالغ (108) درجة، هذا يعني أن أفراد عينة الدراسة نهائيًا لم يتداولوا العملات المشفرة في الأقليم .

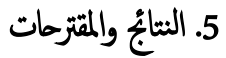

1.5 يتناول المحور الحالي الأستنتاجات التي توصلت اليها الدراسة على صعيد الجانب

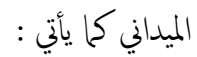
اوضحت نتائج التحليل الأحصـائي نسـبة (83.1\%) من الأفراد المبحوثين للأقليم ليس لديهم اي معرفة مالية بالية العمل بالعملات المشـــفرة وانواعها وكيفية أستخدامحا وطريقة تداولها . اوضحت نتائح التحليل نسـبة (16.9) من الافراد المبحوثين للأقليم لدهم معرفة مالية بالعملات المشـفرة ولكن تباينت واختلفت آراء عينة الدراســة

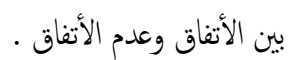
اتقفت عينة الدراسـة بنسـبة عالية من الافراد الذين لديهم معرفة مالية بأن عملية التعدين لها تأثير ســلبي على آلية التداول وذلك لصـعوبها وتكاليفها

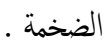
اتنقت عينة الدراسـة بنسـة عالية من الافراد الذين لديهم معرفة مالية بأن المتغيرات الأقتصادية والاوضاع السياسية وسياسة النقدية للدولة لها تأثير

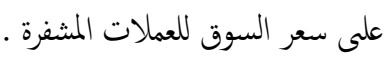
تبين من نتائج التحليل نسبة (100\%) عدم تداول العملات المشفرة للعينة المبحوثة سواءاً كانت الطريقة المباشرة او غير مباشرة.

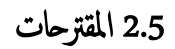
متابعة لما تقدم من أســتنتاجات يعرض المورر الحالي عدداً من المقترحات التي وجدت الباحثة أنها مناسبة وفق الآتي : توصـية منظمات الأعمال وخاصـة الشر_كات تبادل العملات بمثابعة تداول العملات المشفرة وأصـدار النشرات التوعوية بشكل مسيتمر لمستخدميها لمسـاعدتهم في أسـتخداهما بشـكل صحيح وآمن وبعيد عن الخخاطر من اجل حايتهم من النصب والأحتيال . 


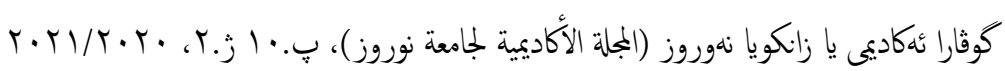

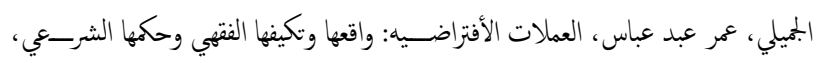

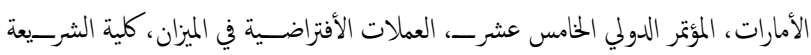

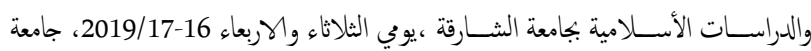
الشارقة، قاعة الزهراء، مبنى wZ، الساعة العاشرة صباحاً.

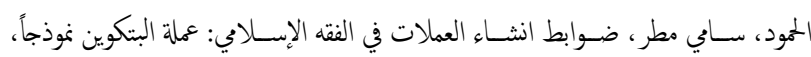

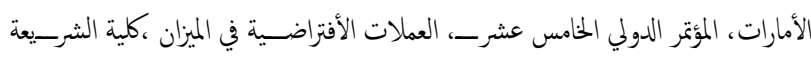

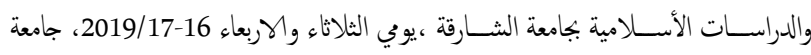
الشارقة، قاعة الزهراء، مبنى wZ، الساعة العاشرة صباحاً.

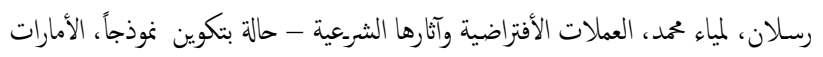

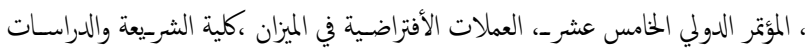

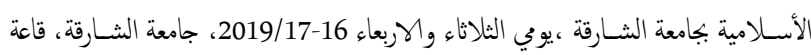
الزهراء، مبنى WZ، الساعة العاشرة صباحاً.

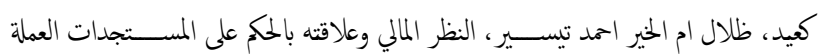

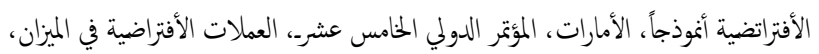

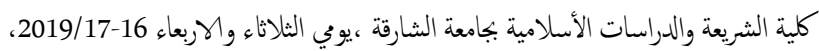
جامعة الشارقة، قاعة الزهراء، مبنى wz، الساعة العاشرة صباحاً.

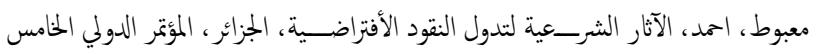

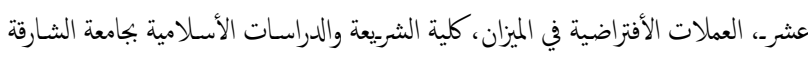

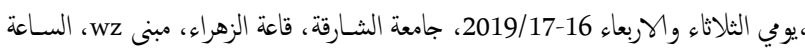
العاشرة صباحاً.

المهداوي والعيسـاوي، علي احمد واسـاعيا كاظم، أبعاد العملة أفنزاضـية، الأمارات، المؤتمر

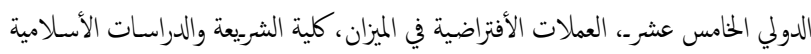

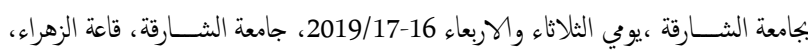

$$
\text { مبنى Wz، الساعة العاشرة صباحاً. }
$$

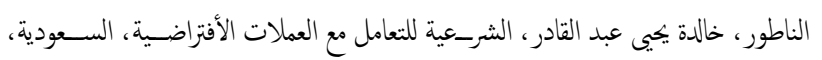

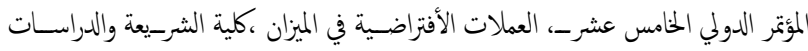

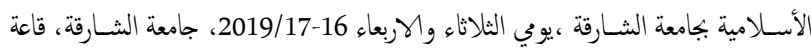

$$
\text { الزهراء، مبنى WZ، الساعة العاشرة صباحاً. }
$$

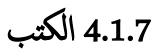

1. شـافي، نادر عبدالعزيز، (2007)، المصـارف والنقود الألكترونية، المؤسـســة الحديثة

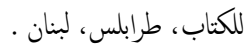

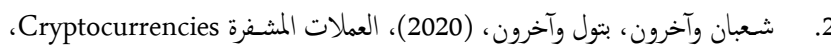

$$
\text { البنك المركزي الأردني : دائرة الإشراف والرقابة على نظام المدفوعات الوطني . }
$$

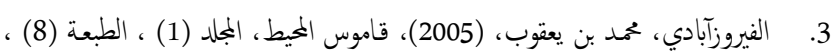

$$
2.7 \text { المصادر الأجنبية الناشئة الرسالة . }
$$

\subsubsection{Scientific Thesis \& Dissertation}

1. Erdugan Riza,(2012), The Effect of Economic Factors on The Performance of The Australian Stock Market, Thesis Degree Of Doctor, Faculty Of Business And Law, University Melbourne.

2. Janicki Jan,(2019), The Macroeconomic Determinants Of Cryptocurrencies' Returns, Dissertation Submitted As Partial Requirement For The Conferral Of Master In Economics, Instituto Universitario De Lisboa..

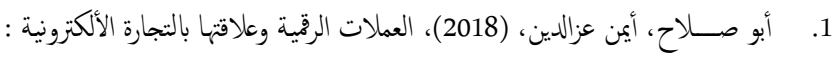

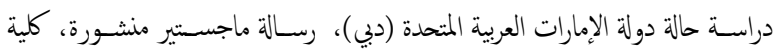

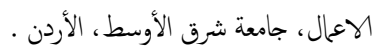

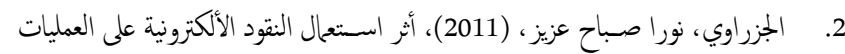

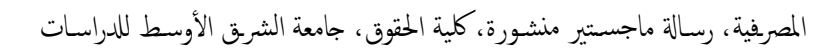

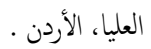

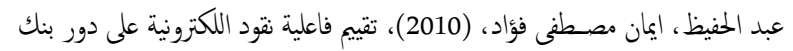

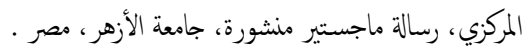
.5

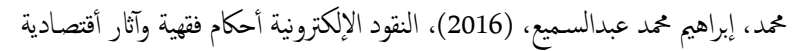

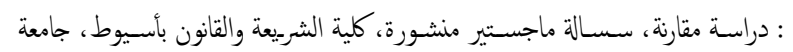

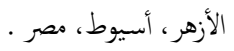

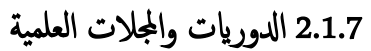

\section{6}

1. احمد وآخرون، منير ماهر وآخرون، (2018)، الكفاءة الأقتصادية للعملات الأفتراضية

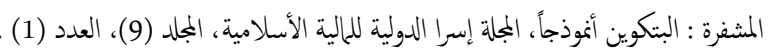

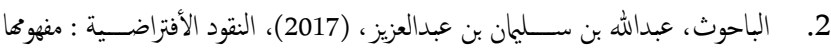

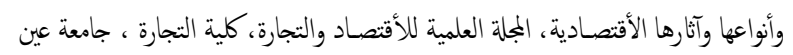

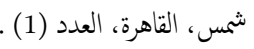

البجاري والأمد، حلا سامي خضير وعمار شهاب احمد، (2018)، تقييم محافظ الأوراق .3

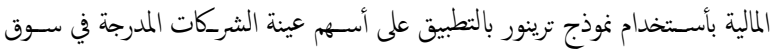

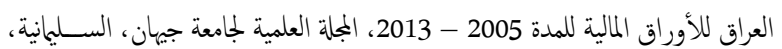

المجلد (1)، العدد (1)

4. حامد، محي الدين، (12014)، عملة البتكوين الكترونية، بنك فيصل الأسلامي السوداني، (1)،

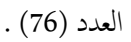

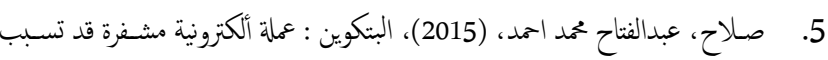

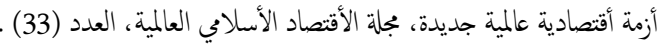

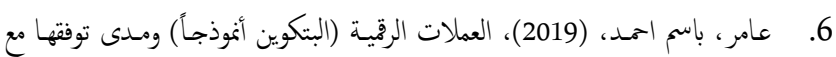

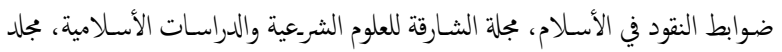

(16)، العدد(1) . (16)

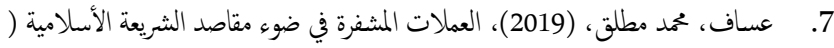

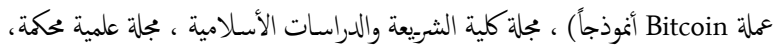
قطر، المجلد (36)، العدد (2) . 8. الكناني، عايد كريم، (2014)، معوقات البحث العلمي كما يراها أعضاء هيأة التدريس في

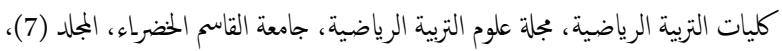

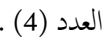
3.1.7 المؤمرات

1. ابو حسين، أسامة اسعد، الحكم الشريعي للتعامل بالعملات الأفتراضية ، الأمارات، المؤتمر

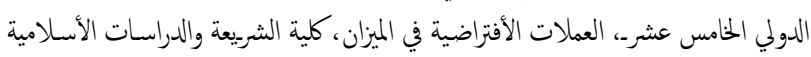

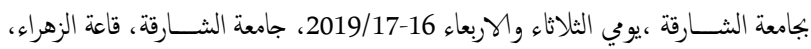
مبنى Wz، الساعة العاشرة صباحاً.

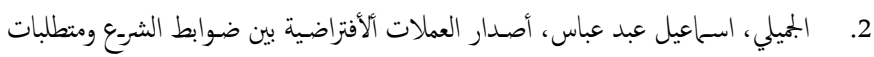

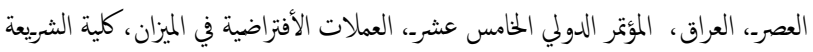

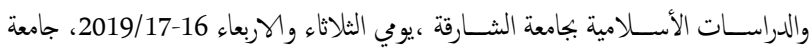
الشارقة، قاعة الزهراء، مبنى wz، الساعة العاشرة صباحاً. 


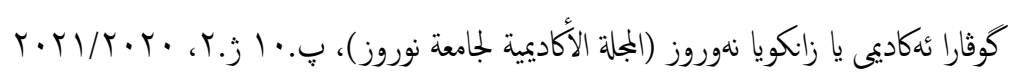

9. Holovatiuk Olha, (2020), Cryptocurrencies as an asset class in portfolio optimization, Central European Economic Journal, 7(54), 33-55. ISSN: 2543-6821 (online), link to this article: https://doi.org/10.2478/ceej-2020-0004.

10. Khazal Aras, A. Krogstad Erlend, B. G. Nordstrøm Frans,(2018), An Analysis of Bitcoin's Price Dynamics, Journal of Risk Financial Management. 2018, 11, 63 Accepted: 11 October 2018; Published: 15 October 2018.

11. Krishnan Hari R., Saketh Y. Sai and Tej Vaibhav M Venkata, (2015), Cryptocurrency Mining - Transition to Cloud, (IJACSA) International Journal of Advanced Computer Science and Applications, Vol. 6, No. 9, 2015

12. Minaudo Massimiliano,(2018), New Digital Currency and Technological Transformation, Cobanoglu and Corbaci Advances in Global Business and Economics: Volume 1.

13. Olena Parshina \& Yurii Savchenko\& Bogdana Polyanovs 'Ka,(2019), Economic Security, Scientific Bulletin Of The Dnipropetrovsk State University Of Internal Affairs. 2019. Special Issue № 1, Doi: 10.31733/2078-3566-2019-5-243-249.

14. Olive,Mario Arias,Borondo Jorge Pelegrin And Clavero Gustavo Matisa, (2019), Variable Influencing Cryptocurrency Use : A Technology Acceptance Model In Spain, Fpsyg.2019.0047510.3389, Orginal Research .

15. Ozyesil Mustafa ,(2019), Is Bitcoin Becoming An Alternative Investment Option For Turkey? A Comparative Econometric Investigation Of The Interaction Between Crypto Currencies, Reserch Journal of Business and Management, ISSN 2184-6689, Volume 6, Issue: 01.

16. Teker, Teker \& Ozyesil, Dilek, Suat, \& Mustafa , (2019), Determinants Of Cryptocurrency Price Movements, 14th PARIS International Conference On Marketing, Economics, Education And Interdisciplinary, ISBN 978-93-86878-32-8, Paris (France) Nov. 12-14, 2019

17. Panos Georgios ,Karkkainen Tatja \& Atkinson Adele,(2020), Financial Literacy and Attitudes to Cryptocurrencies, JEL Classification: B26; D18; E41; G11; G53; Electronic copy available at: https://ssrn.com/abstract=3482083

18. Yhlas, Sovbetov, (2018), Factors Influencing Cryptocurrency Prices: Evidence from Bitcoin, Ethereum, Dash, Litcoin, and Monero, Journal of Economics and Financial Analysis, Vol:2, No:2 , MPRA Paper No. 85036, posted 18 Mar 2018 05:00 UTC.

\subsubsection{Conferences}

1. Ghimire Suman and Selvaraj Henry, (2018), A Survey on Bitcoin Cryptocurrency and its Mining, Conference Paper - December
3. Vieira P. J. M. (2017). Price Analysis of Bitcoin : Volatility, Key Drivers and Evolution. Master Thesis in Finance, FEP, Universidade do Porto.

4. Yanardag Nilay, (2019), Cryptocurrency Investment Decisions and Behavioral Bias Effectm, Master Thesis, Financial Economics Master's Degree PROGRAM, Enstitute Of Social Science, Istanbul Bilgi University

\subsubsection{Journals \& periodical}

1. Abramov Alexander, Radygin Alexander \& Chernova Maria ,(2015), Long-Term Portfolio Investments: New Insight Into Return And Risk, Russian Journal Of Economics 1 (2015) 273293, Russian Journal Economics, Russian Presidential Academy Of National Economy And Public Administration, Moscow, Russiab Gaidar Institute For Economic Policy, Moscow, Russia.

2. Athanasios Andrikopoulos, Robert, S. Hudson, Saeed Akbar, Darius Saftoiu,(2018), Cryptocurrencies meet inflation theory, JEL Classification: $\quad$ E31, $\quad$ G12, $\quad$ G14, $\quad$ G15 https://www.researchgate.net/publication/327474415.

3. Berentsen Aleksander \& Schär Fabian,(2018), A Short Introduction To The World Of Cryptocurrencies, Federal Reserve Bank Of St. Louis Review, First Quarter 2018, 100(1), Pp. 116.Https://Doi.Org/10.20955/R.2018.1-16, (JEL G23, E50, E59).

4. Bohme Rainer \& Nicolas Christin,Benjamin Edelman, \& Tyler Moore,(2015), Bitcoin: Economics, Technology, and Governancet, Journal of Economic Perspectives-Volume 29, Number 2,Spring 2015.

5. Chiu Jonathan and Koeppl V. Thorsten, (2018), The Economics of Cryptocurrencies - Bitcoin and Beyond, JEL Classi_cation: E4, E5, L5, Bank of Canada, 234 Wellington St, Ottawa, ON K1A 0H9, Canada (e-mail: jchiu@bankofcanada.ca).

6. Ciaian, P., Rajcaniova, M., \& Kancs, d'Artis. (2018). Virtual relationships: Short- and long-run evidence from Bitcoin and Altcoin markets. Journal of International Financial Markets, Institutions and Money, 173-195. https://doi.org/10.1016/j.intfin.2017.11.001.

7. Clements Ryan, (2018), Assessing The Evolution Of Cryptocurrency: Demand Factors, Latent Value, And Regulatory Developments Michigan Business \& Entrepreneurial Law Review. REV. 73m Volume 8 Issue 1.

8. Cocco Luisanna And Marchesi Michele,(2016), Modeling And Simulation Of The Economics Of Mining In The Bitcoin Market, Department Of Electric And Electronic Engineering, University Of Cagliari, 09123 Cagliari, Italy, Economics Of Bitcoin Mining, PLOS ONE DOI:10.1371/Journal.Pone.0164603 Luisanna.Cocco@Diee.Unica.It. . 


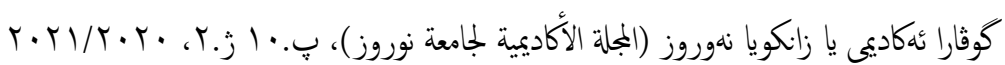

Discussion Paper 2018-44, International Research Training Group 1792,High Dimensional Nonstationary Time Series, http://irtg1792.hu-berlin.de,ISSN 2568-5619.

10. Houben Robby and Snyers Alexander,(2018), Cryptocurrencies and blockchain, Legal context and implications for financial crime, money laundering and tax evasion, IPOL Policy Department for Economic, Scientific and Quality of Life Policies, DirectorateGeneral for Internal Policies, PE 619.024, STUDY Requested by the TAX3 committee.

11. Ibrahim \& Almansour, Halima \&Bashar Yaser, (2020), Cryptocurrency Market: Digital Currency, https://www.researchgate.net/publication/339443427.

12. International Bank for Reconstruction and DevelopmentThe World Bank, Cryptocurrencies and Blockchain, 2018 H Street NW, Washington, DC 20433Telephone: 202-473-1000; Internet: www.worldbank.org2018: 44, Office of the Chief Economist.

13. Jankov Aljosa,(2017), The prospects of Bitcoin as a driver of economic changes ,School of Business and Management, Andrzej Kraslawski.

14. Kampl Alex, (2014), Analysis of Large-Scale Bitcoin Mining Operations (or how Bitcoin miners make \$845 Million a Year, Introduction of the Modular, 1.2MW Bitcoin Mining Container For inexpensive, efficient and rapid cluster deploymenttech@allied-control.com

15. Maire M. Bruno Le, (2019), Digital Currencies, An exploration into technology and money Minister of Economy, Jean-Pierre Landau with Alban Genais.

16. Perkins W. David ,(2020),Cryptocurrency: The Economics Of Money And Selected Policy Issues, Congressional Research Service,Information The Legislative Debate Since Issues. Https://Crsreports.Congress.Gov, R45427.

17. Rodricks, Matthew Robert, (2018), Statistical Determinants of Bitcoin, A Major Qualifying Project submitted to the faculty of Worcester Polytechnic Institute to fulfill the requirements of a Degree in Bachelor of Science in Business, Worcester Polytechnic Institute, Digital WPI, Major Qualifying Projects, https://digitalcommons.wpi.edu/mqp-all.

18. Thornton Grant, (2016), Digital, Virtual and Cryptocurrencies: Issues and Accounting Risks,Grant Thornton International Ltd. Available At: hptt://www.grantthoronton.global.com.

19. Tiwari, A. K., Jana, R. K., Das, D., \& Roubaud, D. (2018). Informational efficiency of Bitcoin-An extension. Economics Letters, 163: $106-109$. https://doi.org/10.1016/j.econlet.2017.12.006.
2018, University of Nevada, Las Vegas https://www.researchgate.net/publication/331040157.

2. Jeong Hoon Oh, (2018), Cryptocurrency and "Kimchi Premium", Forex Market with Cryptocurrency and "Kimchi Premium", Conference Paper ,The 22nd conference of the International Telecommunication Association (ITS): "Beyond Borders: Business, Policy and Society Challenges", Seoul, Korea, 24-27 June 2018 Presented in cooperation with: The International Telecommunication Association (ITS), Leibniz Information Center for Economics.

\subsubsection{Working Papers}

1. Aggarwal Gourang, Patel Vimal, Varshney Gaurav and Oostman Kimberly,(2019), Understanding Social Factors Affecting The Cryptocurrency Market, University of New Mexico Albuquerque .arXiv:1901.06245v1 [cs.CY] 13 Jan 2019,

2. Balcilar, M., Bouri, E., Gupta, R., \& Roubaud, D. (2017). Can volume predict Bitcoinreturns and volatility? A quantiles-based approach. Economic Modelling, 64: 74-81. https://doi.org/10.1016/j.econmod.2017.03.019.

3. Bartram Söhnke M. \& Dufey Günter, (2016), International Portfolio Investment: Theory, Evidence, and Institutional Framework, Finance Markets Institutions \& Instruments, JEL Classification: G15, G11, F31. https://www.researchgate.net/publication/227715964.

4. Baur, D. G., Dimpfl, T., \& Kuck, K. (2018). Bitcoin, gold and the US dollar - Areplication and extension. Finance Research Letters, 25: 103-110.https://doi.org/10.1016/j.frl.2017.10.012.

5. Cocco Luisanna, Tonelli Roberto and Marchesi Michele,(2019), An Agent Based Model to Analyze the Bitcoin Mining Activity and a Comparison with the Gold Mining Industry, 1 Department of Electric and Electronic Engineering, University of Cagliari, 09123 Cagliari, Italy, Future Internet 2019, 11, 8; doi:10.3390/fi11010008.

6. Dorje C. Brody, Lane P. Hughston and Bernhard K. Meister, (2019), Theory of Cryptocurrency Interest Rates, University of London, New Cross, London SE14 6NW, UK rXiv:1904.05472v1 [q-fin.MF] $10 \quad$ Apr 2019.https://www.researchgate.net/publication/332368725.

7. Dyhrberg, A. H. (2016). Bitcoin, Gold And The Dollar - A GARCH Volatility Analysis. Finance Research Letters, 16: 85-92. https://doi.org/10.1016/j.frl.2015.10.008.

8. Grewall, Carr \& V. Marshall, (2016). Blockchain, Enigma, Paradox, Opportunity, Available At: https://www2.deloitte.com.

9. Härdle Wolfgang Karl, Campbell R. Harvey \& Raphael C. G. Reule ,(2019), Understanding Cryptocurrencies, IRTG 1792 


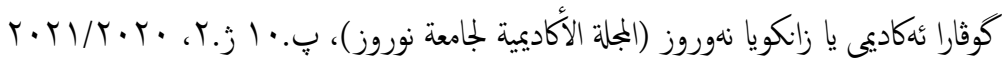

20. Vogel Marius,(2019), The Value Determinants of Cryptocurrencies, Lucerne University of Applied Sciences and Arts https://www.researchgate.net/publication/336209178.

21. Yaish Aviv \& Zohar Aviv,(2020), Pricing ASICs for Cryptocurrency Mining, arXiv:2002.11064v1 [cs.CR] 18 Feb 2020, The Hebrew University of Jerusalem, faviv.yaish, avivzg@mail.huji.ac.il.

22. Yermack, D. (2015). Is Bitcoin a Real Currency? An Economic Appraisal. D. K. C.Lee (ed.), in The Handbook of Digital Currency: Bitcoin, Innovation, Financial Instruments, and Big Data. Elsevier Inc., pp. 31-44. https://doi.org/10.1016/B978-0-12802117-0.00002-3.

23. Zimmerman Peter, (2020), Blockchain structure and cryptocurrency prices, Staff Working Paper No. 855, ISSN 17499135, en updates@bankofengland.co.uk.

\subsubsection{Website}

1. https://coinmarketcap.com/currencies/bitcoin/historical-data/ 